\title{
Endometrial epithelial cell apoptosis is inhibited via a ciR8073- miR181a-neurotensis pathway during embryo implantation
}

\author{
Lei Zhang ${ }^{1, *}$, Xiaorui Liu ${ }^{1, *}$, Yuexia Liu ${ }^{1}$, Xingna Ma ${ }^{1}$, Yue Zhang ${ }^{1}$, Xiaoyan Du ${ }^{1}$, \\ Junze Liu ${ }^{1}$, Zhanqin Zhou ${ }^{1}$, Yuxuan Song ${ }^{1}$ and Binyun Cao $^{1}$ \\ ${ }^{1}$ College of Animal Science and Technology, Northwest A\&F University, Yangling, Shaanxi 712100, P.R. China \\ *These authors have contributed equally to this work \\ Correspondence to: Yuxuan Song, email: yuxuan_song2016@163.com \\ Binyun Cao, email: binyun_cao@163.com \\ Keywords: receptive endometrium; ceRNA; ciR8073-miR181a-NTS; endometrial epithelial cells (EECs); dairy goats \\ Received: August 08, $2017 \quad$ Accepted: December 05, $2017 \quad$ Published: January 16, 2018 \\ Copyright: Zhang et al. This is an open-access article distributed under the terms of the Creative Commons Attribution License 3.0 \\ (CC BY 3.0), which permits unrestricted use, distribution, and reproduction in any medium, provided the original author and source \\ are credited.
}

\section{ABSTRACT}

\begin{abstract}
Development of receptive endometrium (RE) from pre-receptive endometrium $(P E)$ is essential for embryo implantation, but the molecular mechanisms are not fully understood. In this study, IncRNA/circRNA-miRNA-mRNA networks were constructed to explore the functions of potential competing endogenous RNAs (ceRNA) during the development of RE in dairy goats. We observed that circRNA8073 (ciR8073) decreased levels of miR-181a by acting as a miRNA sponge. This effect indirectly increased expression of neurotensin in endometrial epithelial cells (EECs). Neurotensin in turn inhibited EEC apoptosis by increasing expression of BCL-2/BAX via the MAPK pathway, and also increased expression leukemia-inhibitory factor, cyclo-oxygenase 2, vascular endothelial growth factor A and homeobox A10. We have thus identified a ciR8073-miR181a-neurotensin pathway in the endometrium of dairy goats. Via this pathway, ciR8073 functions as a ceRNA that sequesters miR-181a, thereby protecting neurotensin transcripts from miR-181a-mediated suppression in EECs.
\end{abstract}

\section{INTRODUCTION}

The establishment of endometrial receptivity is known as the "window of implantation (WOI)", which is a spatially and temporally restricted stage [1]. During this period, the endometrium undergoes pronounced structural and functional changes that prepare it to be receptive to adhesion by the qualified embryo [2,3]. An abnormal receptive endometrium (RE) is one of the major reasons for the failure of embryo transplantation during assisted reproduction with good-quality embryos [4]. Notably, many studies have reported that attaining endometrial receptivity is a complex process involving numerous molecular mediators [1].

It has been speculated that non-coding (nc) RNAs serve as key regulators of gene expression under physiological and pathological conditions $[5,6]$. These include miRNAs, IncRNAs and circRNAs (a class of RNAs derived mostly from non-canonical splicing in which the exon ends are joined to form a loop)
$[7,8]$. NcRNAs are reportedly crucial for a variety of physiological processes, including the establishment of endometrial receptivity [9-11]. A comprehensive forecasting and analysis of the ncRNAs underlying the progression of RE is essential for the development of effective strategies to develop the endometrium receptivity and improve the implantation rate.

LncRNAs and circRNAs contain miRNA response elements (MREs) and compete with mRNAs for MREs. This enables them to act as molecular sponges for miRNAs, and ultimately de-repress miRNA target genes [12] to influence the post-transcriptional regulation. An appealing aspect of the ceRNAs hypothesis provides a pathway for predicting a non-coding function of any uncharacterized RNA transcript by the identification of putative miRNA binding sites. This hypothesis is now supported by experimental evidence for an accumulating number of lncRNAs [13-15] and circRNAs $[16,17]$. For example, IncRNA-MIAT effectively decreases expression of miR-150-5p and target gene 
VEGF [13]; IncRNA-CCAT1 increases Bmi1 expression by competitively sponging miRNA-218-5p [14]; and lncRNA-MD1 acts as a miR-133 and miR-135 sponge [15]. Although the function of most circRNAs remains elusive, at least three circRNAs have been experimentally shown to act as ceRNAs: ciRS-7 (also known as CDR1AS) inhibits miR-7 activity in the central nervous system [17], an Sry-derived circRNA acts as a sponge for miR138 [18], and ciR-ITCH controls the level of itchy E3 ubiquitin protein ligase by sponging miR-7, miR-17 and miR-214 in oesophageal squamous cell carcinoma [19].

The results outlined above suggest a better understanding the ceRNA could provide new insight into the mechanisms of RE. Our findings will help us to better understand the molecular regulation of RE, and may provide essential information in support of further research on the development of endometrial receptivity.

\section{RESULTS}

\section{Differentially expressed miRNA (DEmiR), mRNA (DEmR), IncRNA (DEIncR), and circRNA (DEciR) between the RE and PE in dairy goat}

In our previous study, a total of 145 DEmiRs meeting the designated criteria of " $P$-values $<0.05$ and fold change $>2$ " (Supplementary File 1), including 111 up-regulated and 34 down-regulated DEmiRs in RE compared with PE [10]. Moreover, miR-181a had the high expression level and the level decreased 0.38 -fold in RE compare with PE.

And 810 mRNAs were found to differ significantly between the PE and RE, the full list of DEmR was provided in Supplementary File 2 meeting the designated criteria of " $P$-values $<0.05$ and fold change $>2$ ". NTS mRNA levels decreased 6.08-fold in RE compared with PE in dairy goats, but this result was inconsistent with the previous what reported that NTS remarkably increased in RE [20]. What's more, a total of 668 DElncRs were found and the full list was provided in Supplementary File 3, and TCONS_00376915 (IncR915) was significantly higher in RE compared with PE. There were 334 DEciRs (Supplementary File 4), 77 DEciRs increased and 257 DEciRs down-regulated in the RE compared with PE. It deserved to note that ciR8073 was expressed specifically in the RE with a higher expression level.

\section{miRNAs-binding sites prediction and ceRNA network construction}

To examine the molecular mechanism of ncRNAs involved in the development of RE, all possible interactions of each DEmiR with all DEmRs (Supplementary File 5), DElncRs (Supplementary File 6) and DEciRs (Supplementary File 7) were analyzed using Targetscan and miRanda. As a result, NTS, IncRNA915 (lncR915) and ciR8073 were predicted as a target of miR181a.

Based on the theory of ceRNA, the DEmiRDEmR, DEmiR-DEciR and DEmiR-DElncR pairs were used to construct the ceRNA networks. As shown in Supplementary File 8, 144 DEmiRs shared 239,385 MREs with 305 DEmRs, 105 DEciRs and 887 DElncRs in the ceRNA network. The ceRNA networks were visualized by importing the above interactions into the Cytoscape software to assemble the regulation network. For example, miR-181a shared MREs with 24 DEmRs, 105 DEciRs and 16 DElncRs in the ceRNA network (Figure 1A, Supplementary File 9). It deserved to note that ciR8073
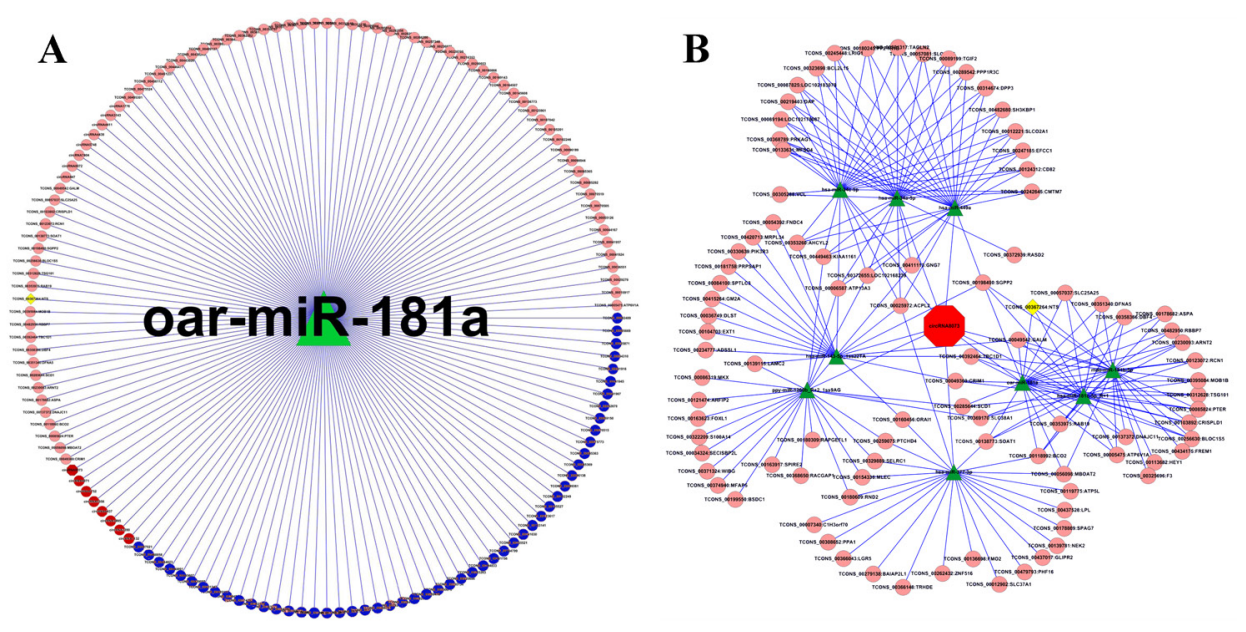

Figure 1: The ceRNA network centered on miR-181a and ciR8073 in endometrium of dairy goats. Note: (A) The differentially expressed targets of miR-181a; (B) the ceRNA network centered on ciR8073. The red hexagon represented ciR8073, and yellow quadrate represented NTS. The green triangle represented miRNAs; red circle represented circRNAs; blue circle represented lncRNAs; pink circle represented circRNAs. 
shared MREs with 9 DEmiRs, including miR-181a (Figure 1B, Supplementary File 10). Indeed, as previously described above, the regulatory role of ncRNAs in RE was very complicated such that an in-depth study should be implement in the future.

To explore the function of the ceRNA network, the interrelated genes within the network were imported to the GO terms and KEGG pathways. Based on GO enrichment analysis, $755 \mathrm{GO}$ functions were found to be the significant component relating the genes in the network, involved in processes such as regulation of cell growth (GO:0001558), cell differentiation (GO:0030154), multicellular organismal development (GO:0007275), cellcell signaling (GO:0007267), apoptosis (GO:0006915), focal adhesion (GO:0005925), metallopeptidase activity (GO:0008237) (Supplementary File 11, Supplementary Figure 1A). Functional pathways analysis demonstrated that the ceRNA network potentially modulated multiple signaling pathways, such as Focal adhesion (ko04510), mTOR signaling pathway (ko04150), Insulin signaling pathway (ko04910), Neurotrophin signaling pathway (ko04722), Apoptosis (ko04210) (Supplementary File 12, Supplementary Figure 1B).

\section{miR-181a and NTS were differentially expressed in the PE and RE}

Stem-loop qRT-PCR was used to validate the expression levels of miR-181a in various tissues of dairy goats, and the results showed it was widely expressed in dairy goats (Figure 2A). The highest expression level was observed in the ovary, followed (in order) by the spleen, oviduct, liver, longissimus dorsi, kidney, lungs, heart and hypothalamus. In the endometrium of dairy goats, the expression level of miR-181a at D15 was lower than that at D5 (Figure 2B). The miRNA in situ hybridization (MISH) assay further showed miR-181a was significantly decreased in RE compared with PE (Table 1), especially in the EECs (Figure 2C-2E).

NTS widely expressed in a series of tissues, and the lowest expression levels were observed in the ovary, increased (in order) by the oviduct, lung, liver, spleen, kidney, longissimus dorsi, hypothalamus, and heart (Figure 3A). What's more, the expression level of NTS increased 195-fold in the endometrium in RE compared to $\mathrm{PE}$ (Figure 3B), which was in accordance with the previous sequencing date [20]. Immunohistochemistry (IHC) results further showed NTS protein increased in RE (Table 2), especially in the EECs (Figure 3C-3F).

\section{The levels of miR-181a and NTS mRNA were response to estrogen (E2) and progesterone (P4) in EECs in vitro}

To investigate the response of miR-181a and NTS levels to sex hormones in EECs in vitro, E2 and P4 were diluted in cell medium to different concentrations. The results showed that miR-181a levels were significantly and concentration-dependently decreased in the presence of $\mathrm{E} 2$ alone, and enhanced in the presence of $\mathrm{P} 4$ alone in EECs (Figure 4A). And the highest levels appeared in 20 $\mathrm{ng} / \mathrm{ml} \mathrm{P} 4$ alone, and lowest level appeared in $100 \mathrm{pg} / \mathrm{ml}$
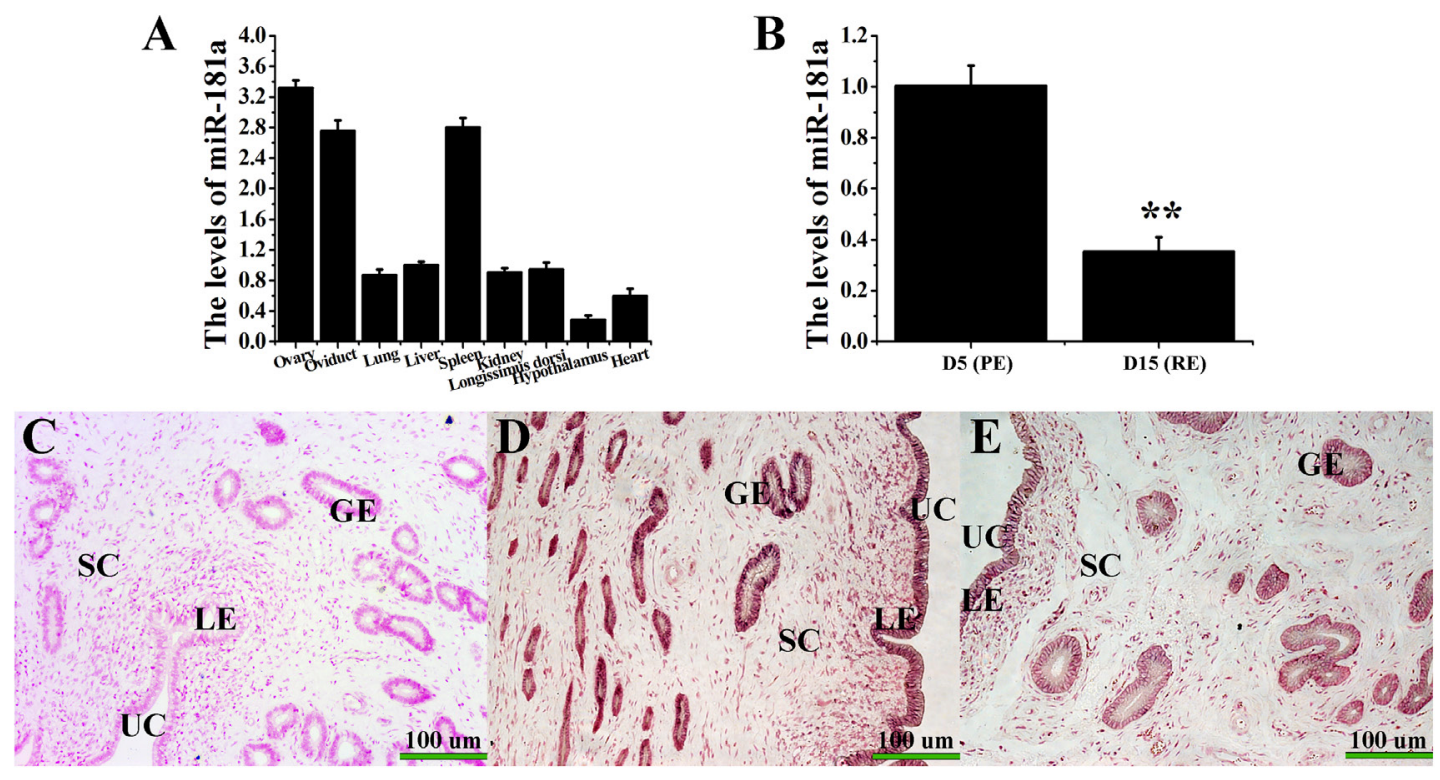

Figure 2: Expression levels of miR-181a in dairy goats. Note: (A) The miR-26a was expressed in various tissues of dairy goats; (B) the miR-181a levels in endometrium in PE and PE; The miR-181a levels were measured by Stem-loop RT-qPCR and normalized to U6, the values were showed as "means \pm SD" $(n=3),{ }^{* *}$ indicates that $p$-value $<0.01$ and ${ }^{*}$ indicates that $p$-value $<0.05$. (C) the NC for in situ hybridization; the miR-181a in the endometrium in PE (D) and RE (E) was detected by in situ hybridization; UC: Uterine cavity; LE: luminal epithelium; GE: glandular epithelium; SC: stroma cell; original magnification $\times 200$; the ruler was $100 \mathrm{um}$. 
Table 1: The MISH staining results of miR-181a in uterus of dairy goats

\begin{tabular}{lccccccc}
\hline \multirow{2}{*}{ Index } & \multicolumn{2}{c}{ Area } & \multicolumn{2}{c}{ Density } & \multicolumn{2}{c}{ IOD } \\
\cline { 2 - 7 } & Mean & Sum & Mean & Sum & Mean & Sum \\
\hline PE (D5) & 758.416 & 1623011 & 0.156 & 333.794 & 210.80 & 451112.63 \\
RE (D15) & $160.026^{*}$ & $189631^{* *}$ & 0.204 & 242.259 & $33.922^{* *}$ & $40197.79^{*}$ \\
\hline
\end{tabular}

Note: MISH was miRNA in situ hybridization, IOD was integrated optical density; ${ }^{*} \mathrm{p}<0.05,{ }^{* *} \mathrm{p}<0.01$.

E2 alone, suggesting that E2 decreased but P4 increased the miR-181a levels in EECs in vitro. What's more, the NTS mRNA levels were detected, and the results showed that E2 and P4 also regulated the NTS mRNA levels but without obvious regularity in EECs in vitro, and the highest levels appeared in a combination of $100 \mathrm{pg} / \mathrm{ml} \mathrm{E} 2$ and $20 \mathrm{ng} / \mathrm{ml} \mathrm{P4}$ (Figure 4B).

\section{miR-181a directly decreased NTS expression in EECs in vitro}

Transfection with miR-181a mimics successfully led to a greater than 168-fold increase, at the same time, miR-181a inhibitors led to 0.39-fold decrease in EECs (Supplementary Figure 2A). To determine whether miR181a directly targets goat NTS through the predicted binding sites in the NTS $3 \mathrm{~S}$ untranslated region (UTR), the psiCHECK ${ }^{\mathrm{TM}}-2$ reporter and mutated plasmids were constructed (Figure 5A). And the luciferase activity of the miR-181a group was significantly lower than that of the $\mathrm{NC}$ group $(\mathrm{p}<0.01)$, but these reduction did not occur with the mutated plasmid (Figure 5B).

The NTS mRNA levels decreased in EECs after transfection with miR-181a mimics $(p<0.05)$, meanwhile, miR-181a inhibitors increased the mRNA levels ( $\mathrm{p}<0.01$, Figure 5C) in EECs in vitro. NTS protein levels were evaluated by performing western blot (WB) analysis, and the quantification of the amount of protein expression was depicted in Figure 5D, what showed the NTS protein significantly decreased in miR-181atransfected cells compared with $\mathrm{NC}(\mathrm{p}<0.05$, Figure 5D). Additionally, miR-181a inhibitors significantly
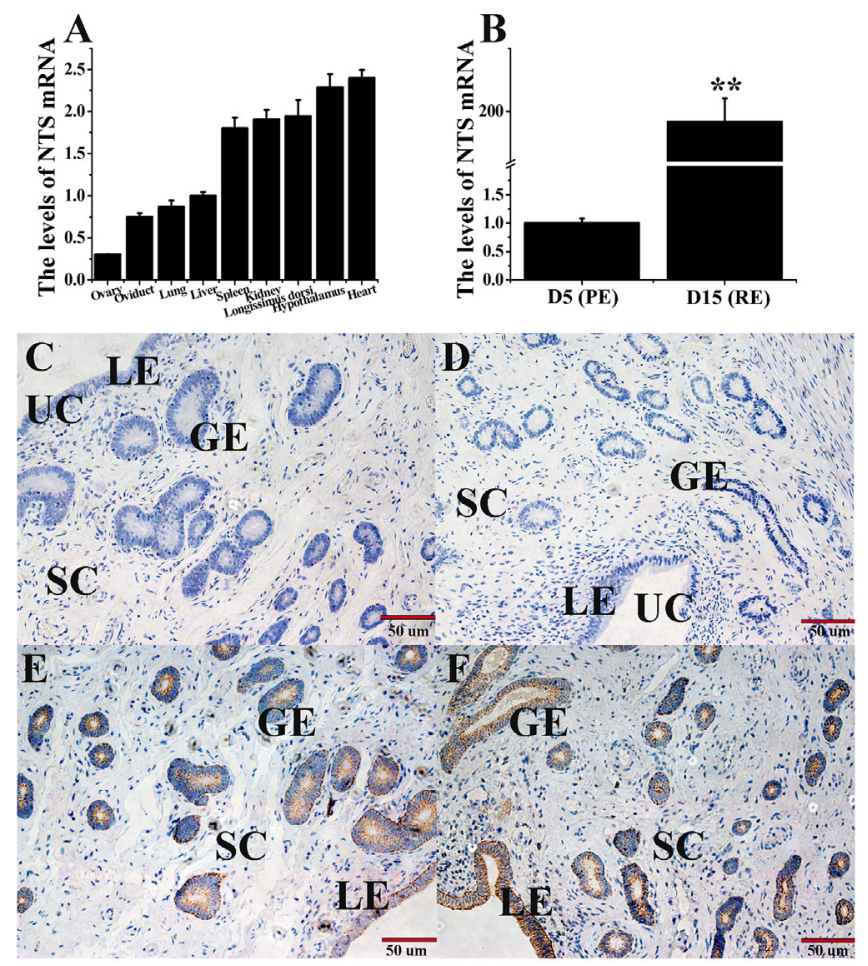

Figure 3: The expression levels of NTS in dairy goats. Note: (A) The NTS was expressed in various tissues of dairy goats; (B) the NTS levels in endometrium in PE and PE; The NTS levels were measured by RT-qPCR and normalized to GAPDH, the values were showed as "means $\pm \mathrm{SD}$ " $(\mathrm{n}=3),{ }^{* *}$ indicates that $\mathrm{p}$-value $<0.01$ and ${ }^{*}$ indicates that $\mathrm{p}$-value $<0.05$. (C, D) the NC for Immunohistochemical staining; NTS levels were detected by Immunohistochemical staining in the endometrium in PE (E) and PE (F); LE, luminal epithelium; GE, glandular epithelium; SC, stroma cell. original magnification $\times 100$; the ruler was 50 um. 
Table 2: The ISH staining results of NTS in uterus of dairy goats

\begin{tabular}{lccccccc}
\hline Index & \multicolumn{2}{c}{ Area } & \multicolumn{2}{c}{ Density } & \multicolumn{2}{c}{ IOD } \\
\cline { 2 - 7 } & Mean & Sum & Mean & Sum & Mean & Sum \\
\hline PE (D5) & 76.71 & 181276 & 0.296 & 699.196 & 21.410 & 50592.813 \\
RE (D15) & 72.699 & 151650 & $0.432^{*}$ & 900.430 & $32.879^{*}$ & $68585.008^{*}$ \\
\hline
\end{tabular}

Note: ISH was in situ hybridization, IOD was integrated optical density; ${ }^{*} \mathrm{p}<0.05$.
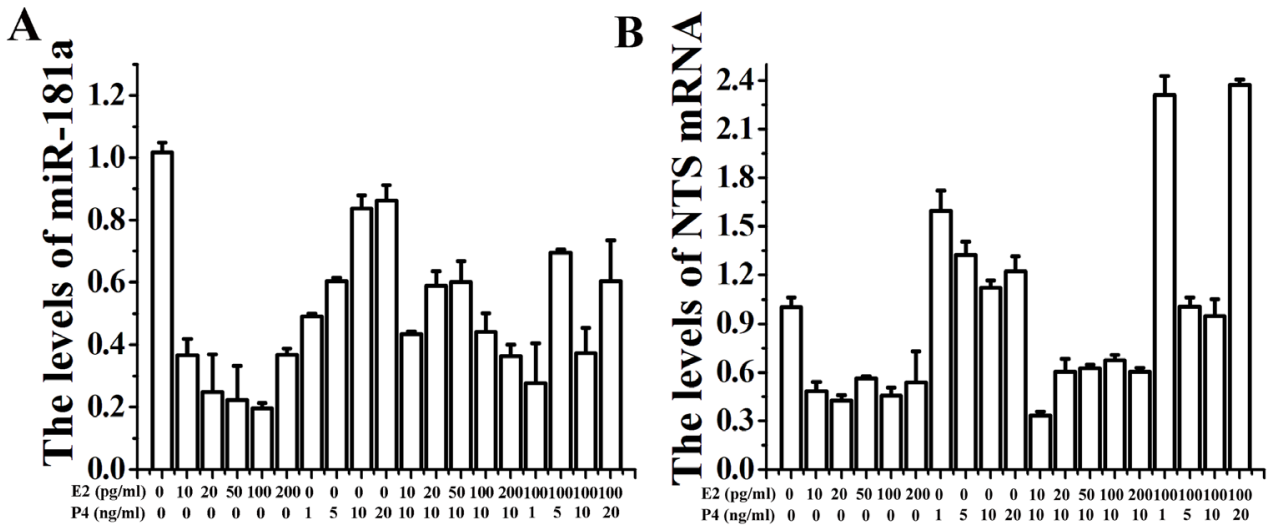

Figure 4: The effect of E2 and P4 on the expression levels of miR-181a and NTS in EECs. Note: (A) the levels of miR-181a; (B) the levels of NTS mRNA. The values were showed as "means \pm SD" $(n=3)$.
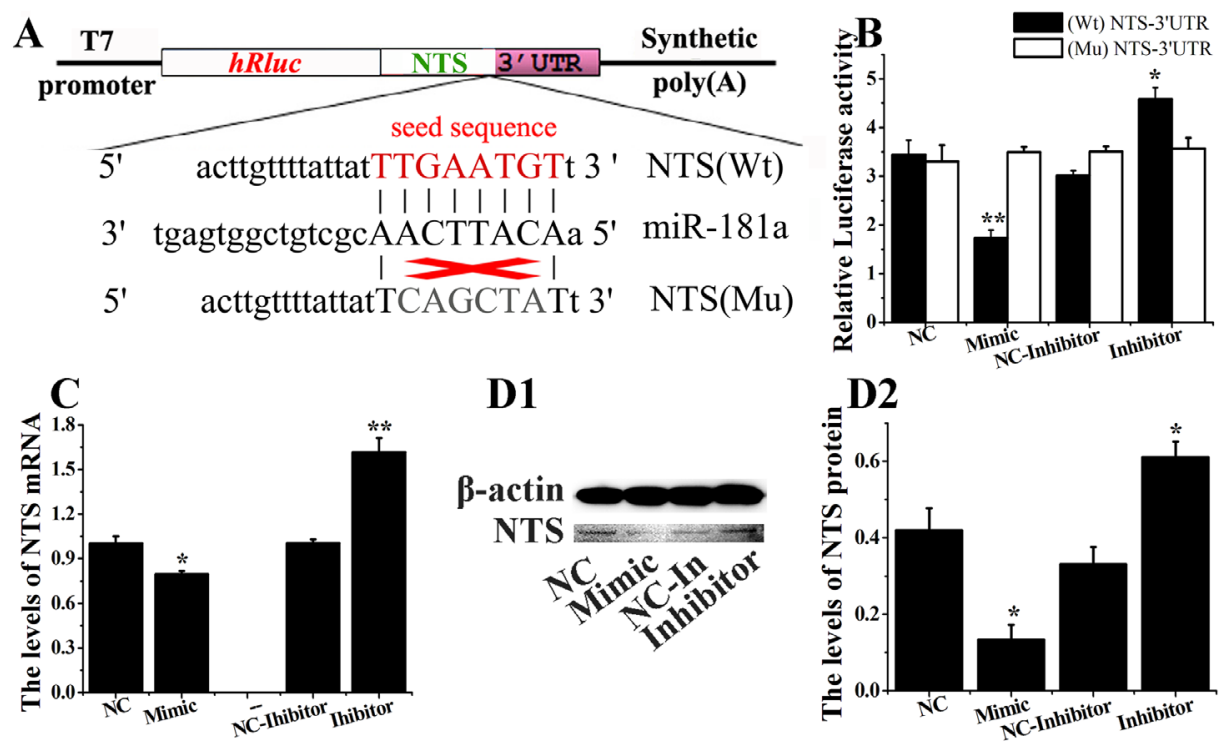

Figure 5: miR-181a down-regulated the expression level of NTS via the $3^{\prime}$ UTR. Note: (A) Schematic diagram illustrating the design of luciferase reporters with the WT- NTS 3' UTR (Wt-NTS) or the site-directed mutant NTS 3' UTR (Mu-NTS). The nucleotides in red represented "seed sequence" of miR-181a, the mutation nucleotides were in gray color. (B) The NTS-3' UTR luciferase reporter vectors were co-transfected with miR-181a mimic (or negative control) into 293T cells, luciferase assay was performed $24 \mathrm{~h}$ after transfection. (C) miR-181a down-regulated the NTS mRNA levels in EEC; NTS mRNA levels were measured by RT-qPCR and normalized to GAPDH. (D) NTS protein levels in EECS were measured by WB, the densitometry was normalized to the $\beta$-actin density from the same lane. Each experiment was repeated three times in triplicate, the results are represented as mean $\pm \mathrm{SD}(\mathrm{n}=3) ;{ }^{*} \mathrm{p}<0.05,{ }^{* * *} \mathrm{p}<0.01$. 
increased the NTS protein in EECs ( $\mathrm{p}<0.05$, Figure 5D). Thus, these results suggested that goat NTS was a target of miR-181a in EECs, what were consistent with our speculation.

\section{miR-181a did not regulate IncR915 in EECs in vitro}

Transfection with pcDNA3.1(+)-lncR915 successfully led to a 89 -fold increase of lncR915 in EECs in vitro $(\mathrm{p}<0.01$, Supplementary Figure $2 \mathrm{~B})$. The psiCHECK ${ }^{\mathrm{TM}}-2$ reporter and mutated plasmids were constructed (Figure 6A), and the luciferase activity of the miR-181a mimics and inhibiters groups did not significantly change compared to the $\mathrm{NC}$ group $(\mathrm{p}>0.05$, Figure $6 \mathrm{~B}$ ). And there was no significant change on the expression levels of lncR915 (Figure 6C) in EECs what were treated with miR-181a mimics and inhibitors. In addition, lncR915 did not change the miR-181a (Figure 6D) and NTS mRNA levels (Figure 6E) in EECs. Thus, these results primarily suggested that miR-181a did not target-regulate lncR915 in the EECs of dairy goats.

\section{ciR8073 served as miR-181a sponge in EECs in vitro}

Transfection with pcDNA3.1(+)-ciR8073 successfully led to a 27.57 -fold increase of ciR8073 compared with NC in EECs in vitro $(\mathrm{p}<0.01$, Supplementary Figure 2C). To determine whether miR- 181a directly targets ciR8073 through the predicted binding sites, the psiCHECK ${ }^{\mathrm{TM}}-2$ reporter and mutated plasmids were constructed (Figure 7A), and the luciferase activity of the miR-181a mimics group was significantly lower than that of the NC group $(\mathrm{p}<0.01)$, and these reductions did not occur with the mutated plasmids $(\mathrm{p}>$ 0.05 , Figure 7B).

Further study indicated that the ciR8073 level decreased significantly when the cells were treated with miR-181a mimics (Figure 7C), and the level also was also decreased by ciR8073 in EECs (Figure 7D). On the contrary, the NTS mRNA significantly increased when the ciR8073 was over-expressed and decreased when ciR8073 was mutated in EECs in vitro (Figure 7E). What's more, the expression levels of NTS were increased by ciR8073, and this increase was counteracted by miR-181a at both the mRNA and protein levels (Figure 7E-7G). Thus, these results suggested that miR-181a targeted ciR8073 what were consistent with our speculation, and verified the accuracy of the network interaction of ciR8073-miR181a-NTS.

\section{miR-181a promoted cell apoptosis of EECs in vitro}

Methyl thiazolyl tetrazolium (MTT) assays showed that miR-181a mimics inhibited the proliferation of EECs (Figure 8A, $\mathrm{p}<0.05$ ), meanwhile, the miR-181a inhibitor promoted the proliferation of EECs $(p<0.05)$. Flow cytometry (FCM) was used to analyze the changes in the
A
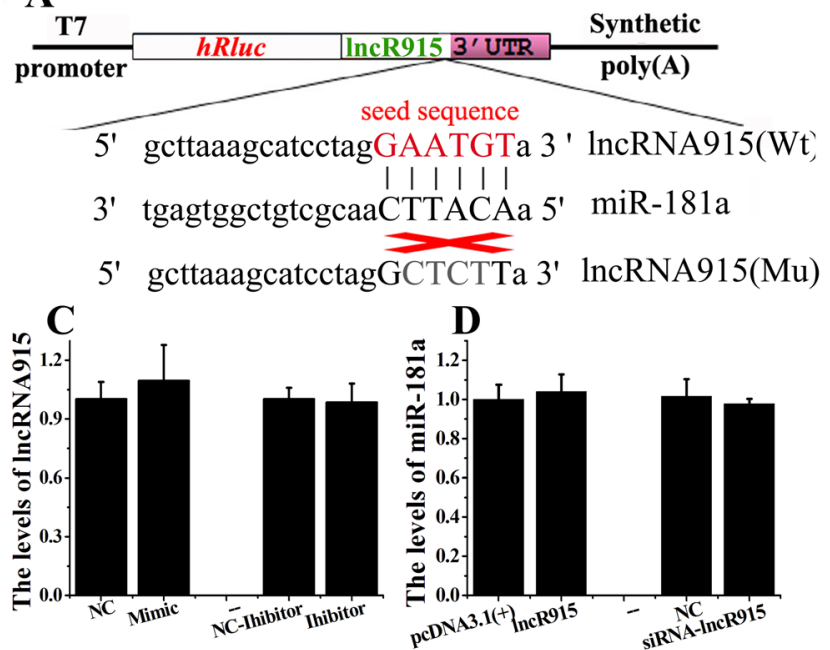
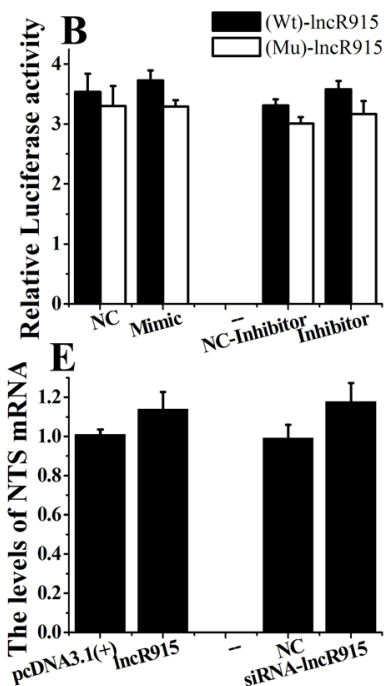

Figure 6: IncR915 was not a target of miR-181a in EECs. Note: (A) Schematic diagram illustrating the design of luciferase reporters with the Wt-lncR915 or the site-directed mutant (Mu-lncR915). The nucleotides in red represented "seed sequence" of miR-181a, the mutation nucleotides were in gray color. (B) The lncR915 luciferase reporter vectors were co-transfected with miR-181a mimic (or negative control) into $293 \mathrm{~T}$ cells, luciferase assay was performed $24 \mathrm{~h}$ after transfection. (C) miR-181a did not regulate the lncR915 levels in EECs; lncR915 levels were measured by RT-qPCR and normalized to GAPDH. (D) lncR915 did not regulate the miR-181a levels in EECs. (E) lncR915 did not regulate the NTS mRNA levels in EECs. Each experiment was repeated three times in triplicate, the results are represented as mean $\pm \mathrm{SD}(\mathrm{n}=3)$. 
cell cycle, and the results showed that the numbers of cells in S-phase was higher in the miR-181a mimics treated EECs that than in NC (Figure 8B, Supplementary Figure 3). Furthermore, an Annexin V-FITC/PI assay combined with FCM was used to detect the effects of miR-181a on cell apoptosis, and the results showed that miR-181a obviously induced the apoptosis of EECs ( $<<0.01$, Figure 8C), meanwhile, the miR-181a inhibitor inhibited the apoptosis ( $\mathrm{p}<0.05$, Supplementary Figure 4).

The BCL-2 expression levels were decreased significantly by miR-181a mimics and increased significantly by inhibitors $(p<0.05$, Figure $8 D)$. On the contrary, miR-181a mimics increased and inhibitors decreased BAX protein levels $(\mathrm{p}<0.05$, Figure 8D). What's more, miR-181a mimics facilitated the phosphorylation JNK ( $\mathrm{p}-\mathrm{JNK}$ ) and increased the levels of P27, miR-181a inhibitors decreased the levels of P27 and PI3K ( $p<0.05$, Figure 8D).

In addition, the changes in LIF, COX2, VEGFA, HOXA10 and osteopontin (OPN) protein levels were investigated in endometrial cells after they were treated with miR-181a mimics or inhibitors. The LIF and COX2 protein levels increased in miR-181a mimics-treated EECs $(\mathrm{p}<0.05)$, and decreased in the inhibitors group $(\mathrm{p}<0.05$, Figure 8D). However, the opposite results were detected on the OPN protein levels, which suggested miR-181a inhibited the OPN in EECs in vitro ( $p<0.05$, Figure $8 D)$.

\section{NTS inhibited cell apoptosis of EECs in vitro}

To further investigate the function of NTS in EECs, an overexpression vector [pcDNA3.1(+)-NTS] was constructed and siRNA-NTS was synthesized, and NTS was overexpressed or inhibited successfully in EECs in vitro (Supplementary Figure 5). In addition, the levels of miR-181a decreased by NTS, and increased by siRNANTS (Supplementary Figure 6), suggested a negative relationship between NTS and miR-181a in EECs.

MTT assay showed that over-expressed NTS markedly promoted $(\mathrm{p}<0.05$, Figure 9A) and siRNA-
A
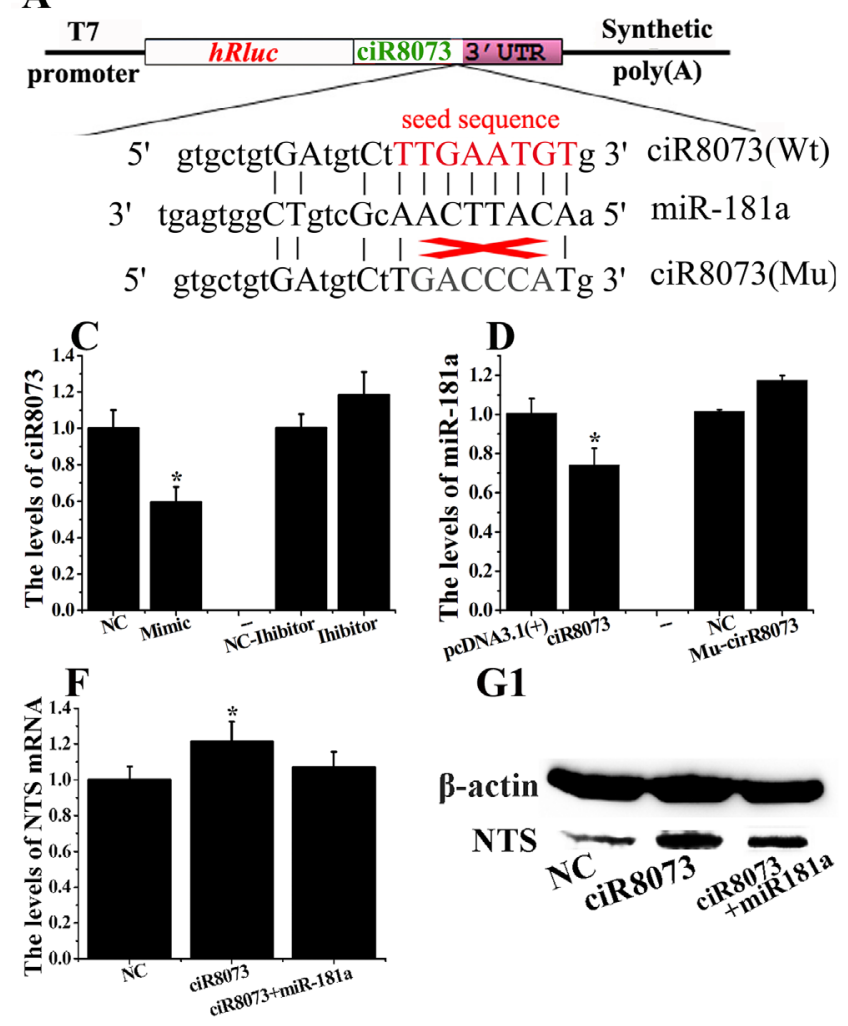

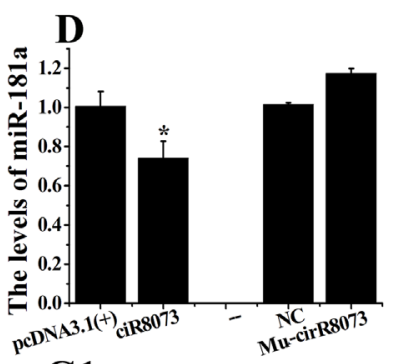

G1

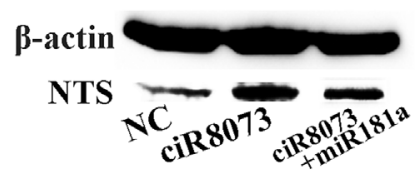

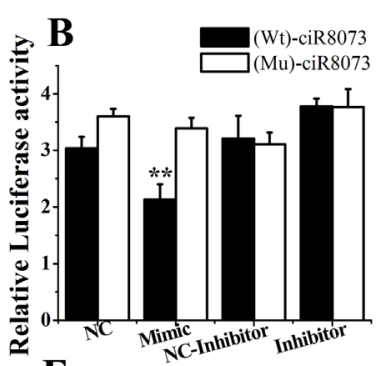
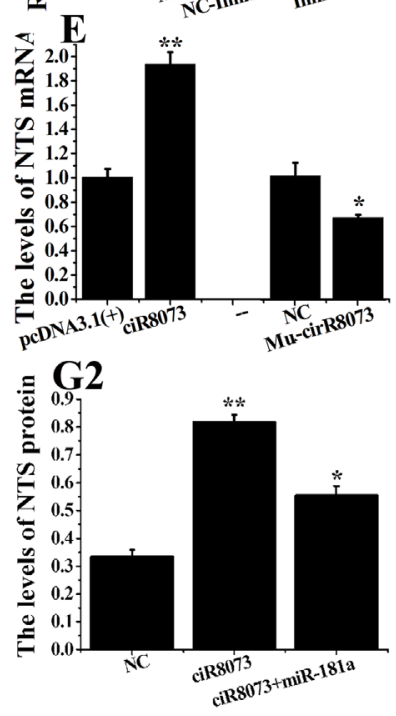

Figure 7: ciR8073 could decrease the levels of miR-181a served as a miRNA sponge in EECs. Note: (A) Schematic diagram illustrating the design of luciferase reporters with the Wt-ciR8073 or the site-directed mutant (Mu-ciR8073). The nucleotides in red represented "seed sequence" of miR-181a, the mutation nucleotides were in gray color. (B) The ciR8073 or its mutation luciferase reporter vectors were co-transfected with miR-181a mimic (or negative control) into $293 \mathrm{~T}$ cells, luciferase assay was performed $24 \mathrm{~h}$ after transfection. (C) miR-181a decreased the ciR8073 levels in EECs, the levels were measured by RT-qPCR and normalized to GAPDH. (D) The miR-181a levels in EECs what were overexpressed or mutational ciR8073 (Mu-ciR8073). (E) The NTS mRNA levels in EECs what were overexpressed or mutational ciR8073 (Mu-ciR8073). (F) The NTS mRNA levels in EECs what were overexpressed ciR8073 and miR181a. (G) NTS protein levels in EECs what were overexpressed ciR8073 and miR-181a, the densitometry was normalized to the $\beta$-actin density from the same lane. Each experiment was repeated three times in triplicate, ${ }^{*} \mathrm{p}<0.05,{ }^{* *} \mathrm{p}<0.01$. 

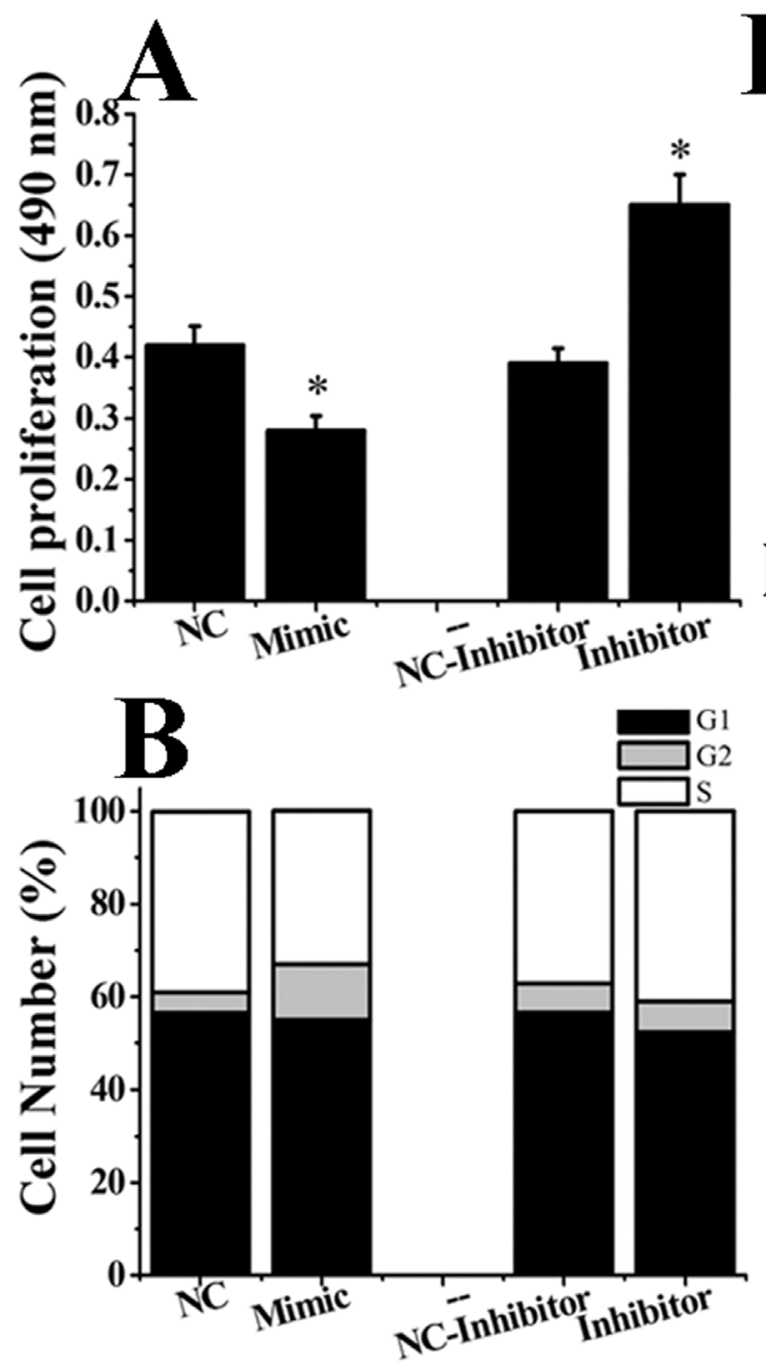

$\beta$-actin

BCL-2

BAX

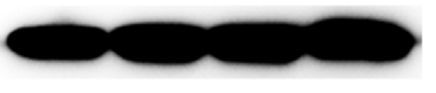

ERK1/2

p-ERK1/2
JNK

p-JNK

$*$

P38
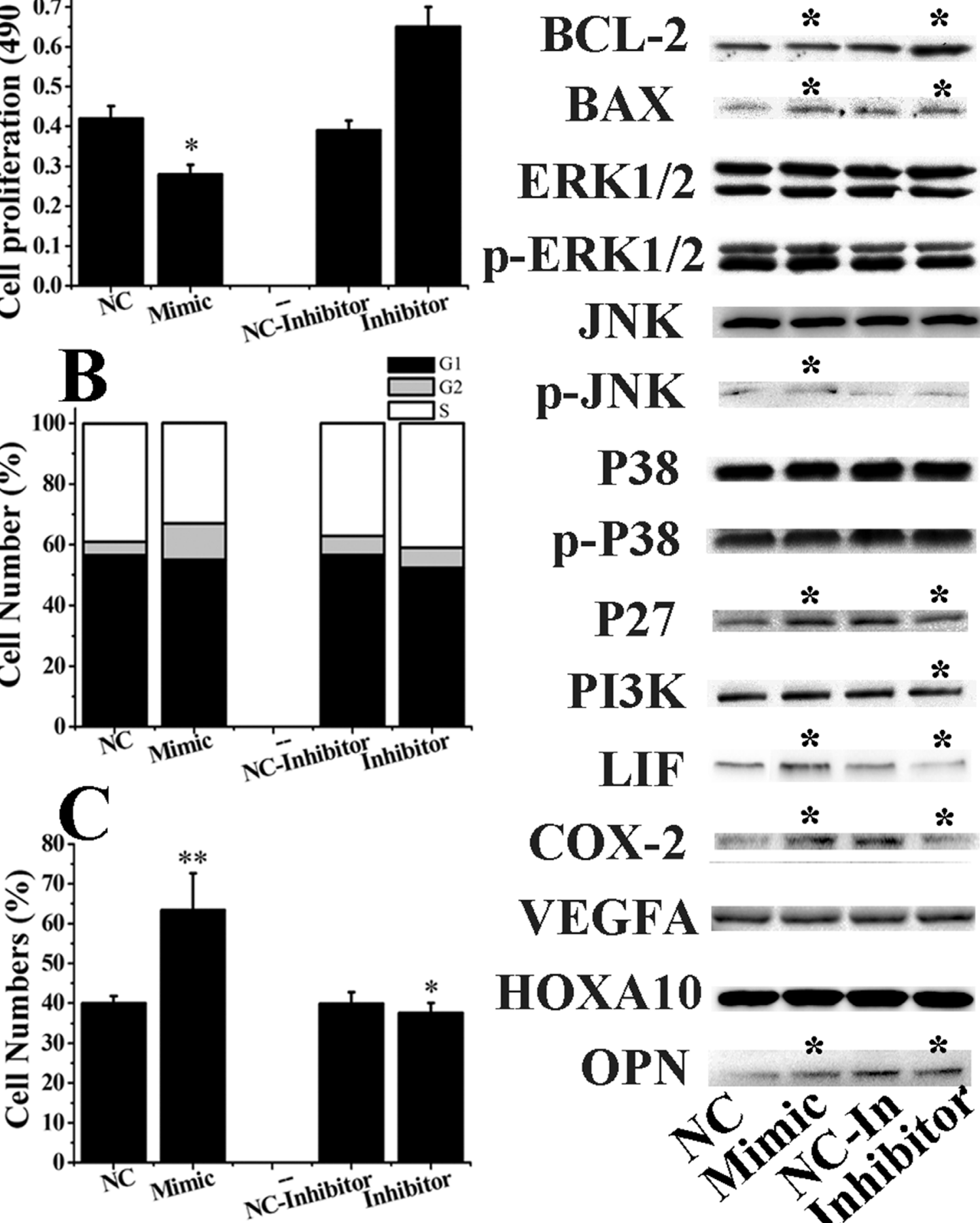

p-P38

P27

$*$

$*$

PI3K *

P131

LIF $-m-$

COX-2 $\quad * x$ min

VEGFA

HOXA10

OPN

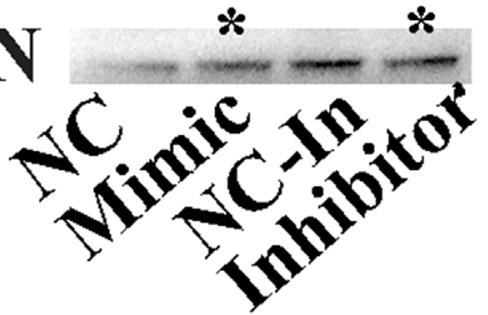

Figure 8: miR-181a promoted EECs apoptosis in vitro. Note: (A) The effect of miR-181a on the proliferation of EECs what were measured by MTT. Values were showed as "means \pm SD" ( $(n=7)$. The cell cycle $(\mathbf{B})$ and apoptosis $(\mathbf{C})$ analysis of EECs what were detected with FCM. (D) The levels of BCL-2, BAX, MAPK pathway proteins, and endometrial receptivity markers (LIF, COX2, VEGFA, HOXA10 and OPN) were measured by WB after the EECs were treated with miR-181a. Densitometry was normalized to the $\beta$-actin density from the same lane, ${ }^{* *}$ indicates that $\mathrm{p}$-value $<0.01$ and ${ }^{*} \mathrm{p}<0.05$. 
NTS significantly inhibited EECs proliferation $(\mathrm{p}<0.05$, Figure 9D). No notably change was observed in the analysis of cell cycle ( $>>0.05$, Figure 9B, 9E), but overexpressed inhibited ( $p<0.05$, Figure $9 C)$ and siRNA-NTS dramatically induced EECs apoptosis $(p<0.05$, Figure 9F; Supplementary Figure 6, Supplementary Figure 7). Thus, some apoptosis-related genes were detected when NTS was overexpressed in EECs, the results showed that NTS increased the protein levels of BCL-2, Caspase-8, FAS, and pleiotrophin (PTN), and decreased BAX and $\mathrm{Sp} 1$ transcription factor (SP1) $(\mathrm{p}<0.05$, Figure 10$)$. In addition, NTS also increased p-ERK1/2, JNK, p-JNK, and p-P38, but decreased the total level of JNK. At the same time, NTS decreased the protein levels of P27 and PI3K $(p<0.05$, Figure 10). What's more, the protein levels of BCL-2 and BAX were also detected in EECs when the NTS was silenced by siRNA-NTS, and the results showed that BCL-2 decreased and BAX increased (Supplementary Figure 9), what were in accordance with the function of miR-181a but contrary to overexpressed NTS in EECs in vitro. All these results suggested that miR-181a induced EECs apoptosis by targeted NTS.

As to the biochemical endometrial receptivity biomarkers, WB analysis showed NTS increased LIF, COX2, VEGFA and HOXA10 ( $<<0.05$, Figure 10). In addition, the endometrial receptivity biomarkers were detected in EECs when the NTS was knockout by siRNANTS, and the results showed siRNA-NTS increased the VEGFA levels in EECs in vitro (Supplementary Figure 10) what needed further studies.

\section{DISCUSSION}

\section{ceRNA network in the endometrium of dairy goats}

Accumulated studies revealed that ceRNAs could serve as post-transcriptional regulators of protein-coding gene expression by decoying miRNAs from other target transcripts, such as lncRNAs, mRNAs, pseudogenes and circRNAs
$[21,22]$. In this study, based on the high-throughput RNAsequencing data, we constructed putative ceRNA networks by integrating lncRNAs, circRNAs, mRNAs and miRNAs in the endometrium of dairy goats. This was the first study to comprehensively identify ncRNAs in the development of RE from $\mathrm{PE}$ by regulatory network analysis.

The mechanism of the ceRNA network was that all types of RNA transcripts could communicate with each other by competing for binding to shared MREs [23]. Some lncRNAs could be considered ceRNAs because they could serve as miRNA "sponges" that inhibit interaction with their miRNA targets in post-transcriptional regulation. Sun established that 51 miRNAs sharing 13,623 MREs with 2260 miRNAs and 82 lncRNAs were involved in the ceRNA networks of cell lines SHEE and SHEEC [24]. In this study, we constructed the DElncR/DEciR-DEmiRDEmR network in the endometrium of dairy goats, and the results showed that 144 DEmiRs shared 239,385 MREs with 305 DEmRs, 105 DEciRs, and 887 DElncRs in the ceRNA networks. It deserved to note that ciR8073 shared MREs with 9 differentially expressed miRNA, including miR-181a. What's more, miR-181a shared MREs with 24 mRNAs, 105 circRNAs and 16 lncRNAs in the ceRNA networks, and NTS mRNA, ciR8073 and lncR915 shared the same MRE of miR-181a.

\section{miR-181a and NTS were regulated by E2/P4 in EECs in vitro}

Because E2 and P4 played leading roles in the uterus of animals, and the concentrations underwent dynamic changes under different physio-pathological conditions [25], we detected the miR-181a and NTS mRNA levels in EECs after the cells were treated with different concentrations of E2 and/or P4, and the data suggested that the levels of miR-181a and NTS mRNA were regulated by $\mathrm{E} 2$ and $\mathrm{P} 4$ in vitro. Further analysis found that miR-181a was widely expressed in different tissues of dairy goats, and the levels decreased in RE compared with PE. Under
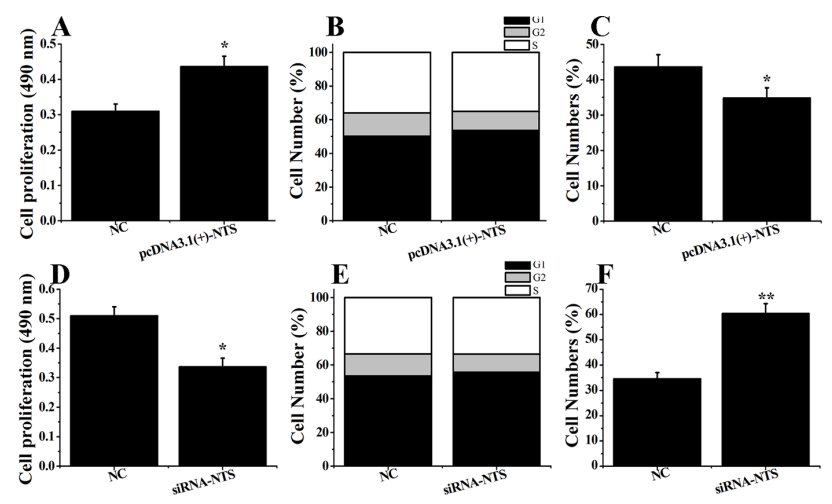

Figure 9: NTS inhibited EECs apoptosis in vitro. Note: (A, D) The effect of NTS on the proliferation of EECs what were measured by MTT. Values were showed as "means \pm SD" $(\mathrm{n}=7)$. The cell cycle $(\mathbf{B}, \mathbf{E})$ and apoptosis $(\mathbf{C}, \mathbf{F})$ analysis of EECs what were detected with FCM. Values were showed as "means \pm SD" $(\mathrm{n}=3)$. ${ }^{* *}$ indicates that $\mathrm{p}$-value $<0.01$ and ${ }^{*}$ indicates that $\mathrm{p}$-value $<0.05$. 


\section{$\beta$-actin}

BCL-2

$* *$

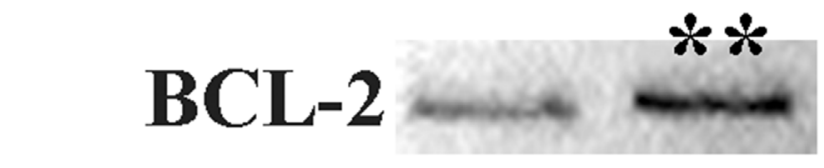

Caspase-8

FAS

PTN $=\frac{*}{*}$

ERK1/2 $=$

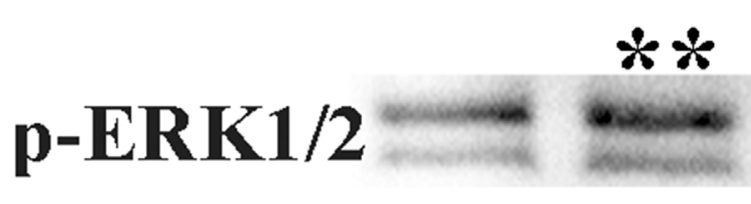

JNK - ***

p n N
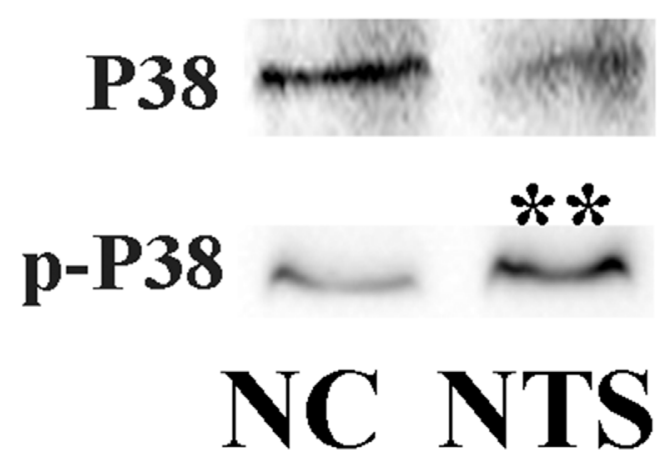

\section{$\beta$-actin}

\section{$* *$ \\ BAX}

$* *$

Caspase-3
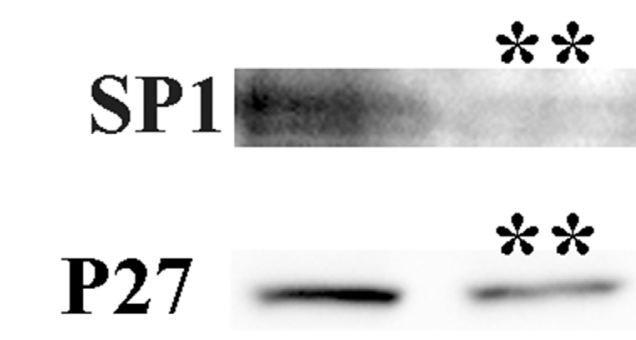

PI3K $-\frac{* *}{*}$
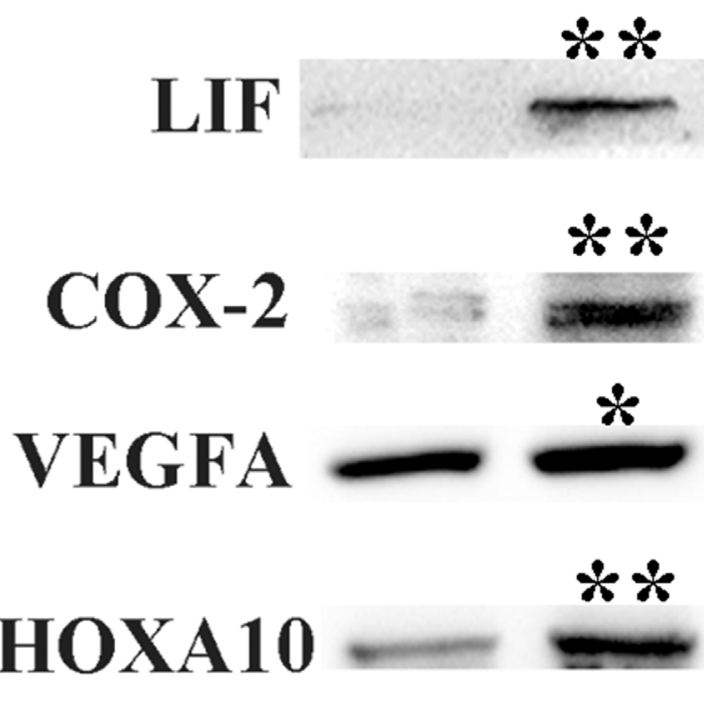

OPN

Figure 10: NTS increased the expression levels of BCL-2/BAX, and increased LIF, COX2, VEGFA and HOXA10 in EECs in vitro. The levels of apoptosis-related proteins (BCL-2, BAX, Caspase-8, Caspase-3, FAS, SP1 and PTN), MAPK pathway proteins, and endometrial receptivity markers (LIF, COX2, VEGFA, HOXA10 and OPN) were measured by WB when the NTS was overexpressed in EECs. Densitometry was normalized to the $\beta$-actin density from the same lane, ${ }^{* *}$ indicates that $\mathrm{p}$-value $<0.01$ and ${ }^{*} \mathrm{p}<0.05$. 
the coordination of E2 and P4, EECs underwent structural and functional changes that established endometrium receptivity [26], which was absolute necessary for successful embryo implantation in all mammals. NTS was also widely expressed in different tissues of dairy goats, and its protein increased in the endometrium in RE compared to PE. Thus, we hypothesized that NTS expression was also regulated by E2 and P4 in the endometrium of dairy goats, and this conjecture was verified in the present study in which we observed a direct regulation of NTS by E2 and $\mathrm{P} 4$ in the EECs in vitro.

\section{miR-181a decreased NTS levels in EECs}

In pigs, miR-181a was found highly expressed on day 15 of gestation, followed by decreased expression on gestational days 26 and 50 in the endometrium during pregnancy [27]. And miR-181a could promote hESCs (human endometrial stromal cells) decidualization-related gene expression and morphological transformation; conversely, inhibition of miR-181a expression compromised hESC decidualization in vitro [28]. NTS was a 13 amino acid peptide, which was biologically active in the central nervous system and in the periphery $[29,30]$. In addition, the higher levels of NTS expressions in the endometrial epithelial and glandular epithelial cells were detected in the bovine endometrium, and more abundant in the endometrium from summer than that from autumn [31]. In this study, miR-181a directly inhibited NTS expression levels at the mRNA and protein levels via its 3' UTR in EECs of dairy goats.

\section{ciR8073 displayed a sponging effect for miR- 181a in EECs}

At present, some lncRNAs and circRNAs had been confirmed as ceRNAs to involve in a variety of physiological functions and pathological processes. For example, lncR-MD1 could control muscle differentiation by liberating the differentiation factors $\mathrm{MEF} 2 \mathrm{C}$ and MAML1 from repression by decoying miR-133 and miR135 [15], and LncR-CHRF regulated cardiac hypertrophy by targeting miR-489 [32]. However, miR-181a did not target lncR915 in EECs of dairy goats, what was inconsistent with our speculation.

Hansen identified a highly expressed ciRS-7, contained more than 70 selectively conserved miRNA target sites, markedly suppressed miR-7 activity, resulting in increased levels of miR-7 targets [16]. What's more, a testis-specific circRNA, sex-determining region Y (Sry), served as a miR-138 sponge suggesting miRNA sponge effects achieved by circRNA formation were a general phenomenon [18]. In EECs of dairy goats, ciR8073 displayed a sponging effect for miR-181a, and the NTS levels significantly increased when the ciR8073 was overexpressed and decreased when ciR8073 was mutated in EECs, suggesting a high degree of endogenous interaction.
This study served as the first, to our knowledge, functional analysis of a naturally expressed circRNA in dairy goats.

\section{miR-181a promoted EECs apoptosis in vitro}

In many mammals, endometrial cells were remodeled by apoptosis and proliferation throughout the estrous cycle [33], and studies had been performed in human [34], mice [35], pig [36] and so on. Furthermore, a coordinated regulation of the embryonic induction of EECs apoptosis was crucial for the embryo to breach the epithelial barrier in vitro [37]. Recently, it had been reported that inhibition of miR-181a might suppress proliferation and promote apoptosis of HeLa and CaSKi cells by modulating the PTEN/Akt/FOXO1 signaling pathway [12]. The balance between BCL-2 and BAX played a key role in determining apoptosis or cell survival [38], and a high BCL-2/BAX ratio made cells resistant to apoptotic stimuli, while a low ratio may predispose them to apoptosis [39]. In the present study, we found lower levels of BCL-2 in the miR-181a-treated EECs, meanwhile, BAX levels increased. Thus, we hypothesized that miR181a plays a role in the regulation of endometrium cell apoptosis via BCL-2/BAX in EECs of dairy goats.

In this study, miR-181a promoted EECs apoptosis and a further study showed that miR-181a decreased the BCL-2 and increased the BAX, what was agree with previous study which reported that miR-181a could direct target BCL-2 in endothelial cells [40]. Furthermore, miR-181a increased p-JNK and P27, and these results suggested that miR-181a might play an important role as an apoptosis-regulating factor by MAPK pathway in EECs of dairy goat.

\section{miR-181a might participate in the formation of endometrial receptivity}

Recently, several morphological and biochemical biomarkers of endometrial receptivity were proposed, including LIF, COX2, VEGFA, HOXA10, and OPN. LIF was seen in the cytoplasm of the EECs [41], and it was widely acknowledged as biochemical markers of receptive endometrium [42]. COX2 was a rate-limiting enzyme for the synthesis of prostaglandin, which was a critical factor for maintaining the uterine environment during early pregnancy [43], and we found that the COX2 protein levels were decreased by the miR-181a in EECs. In addition, VEGFA was expressed in the mouse and rabbit endometrium and likely participated in the increased angiogenesis and vascular permeability necessary for implantation [44, 45]. Evidence suggested that the expression of VEGFA was highly regulated in a temporal and spatial manner at the early stage of implantation [46]. HOXA10, a homeobox-containing transcription factor, expressed cyclically during the menstrual cycle in the endometrium under the influence of steroid hormone, and 
had the highest expression level during WOI [47, 48]. OPN played an important role in endometrial receptivity due to its consistent up-regulation during the WOI [49]. In this study, miR-181a significantly increased LIF and COX2 protein levels but decreased the levels of OPN in EECs in vitro. These results suggested that miR181a might participate in the formation of endometrial receptivity.

\section{NTS promoted EECs proliferation and inhibited apoptosis in vitro}

Previously, much attention was devoted to the function of NTS in cancer [50], or as an important regulator of the fat absorption and obesity [51]. NTS was detected in bovine endometrium, and the expression levels showed difference between the breeding and non-breeding seasons [31]. However, there was no relevant literature on the function of NTS in the formation of endometrial receptivity in dairy goats, and this study was the first one to study the function of NTS in EECs of dairy goats.

At present study, NTS could promote EECs proliferation and inhibit cell apoptosis in vitro. Caspase- 8 was crucial for apoptosis progression, when it was degraded or decreased, resulting in immortality of injured cells and induction of various cancers [52]. Furthermore, several studies had suggested that endometrial cell death was increased by the active Caspase-3 during the estrous cycle [53]. In this study, we found that the NTS did not affect the Caspase- 8 protein levels but increased the Caspase-3 protein levels in EECs. In addition, NTS did not change the protein levels of FAS, what was a critical factor for the progression of extrinsic apoptosis in human endometrum $[34,54]$.

Iwahori reported that SP1 might play a role in DNA repair at damage sites and inhibited cell apoptosis [55], however, silencing SP1 suppressed telomerase activity and promoted apoptosis of SW480 cells line in colorectal carcinoma [56]. In this study, NTS dramatically decreased the SP1 protein levels in EECs, which agreed with the FCM results. PTN was a secreted cytokines that played roles in diverse biology process, such as cell adhesion, migration, survival, growth, and differentiation [57]. In this study, the protein levels of PTN increased with the increase of NTS in EECs. Considering the fact that PTN mainly expressed in the caruncular areas of bovine endometrium [58], it might participate in the proliferation of endometrial cells in dairy goats.

Further investigation revealed that NTS decreased the total levels of MAPK and JNK, but increased phosphorylation levels of MAPK, JNK and P38, meanwhile, it decreased the protein levels of P27 and PI3K in EECs in vitro. Several studies had shown that the ERK1/2 activation usually protected neurons from apoptosis, whereas prolonged activation of $\mathrm{P} 38$ and/or JNK often caused death [59]. Moreover, Chen reported that P27 expression level

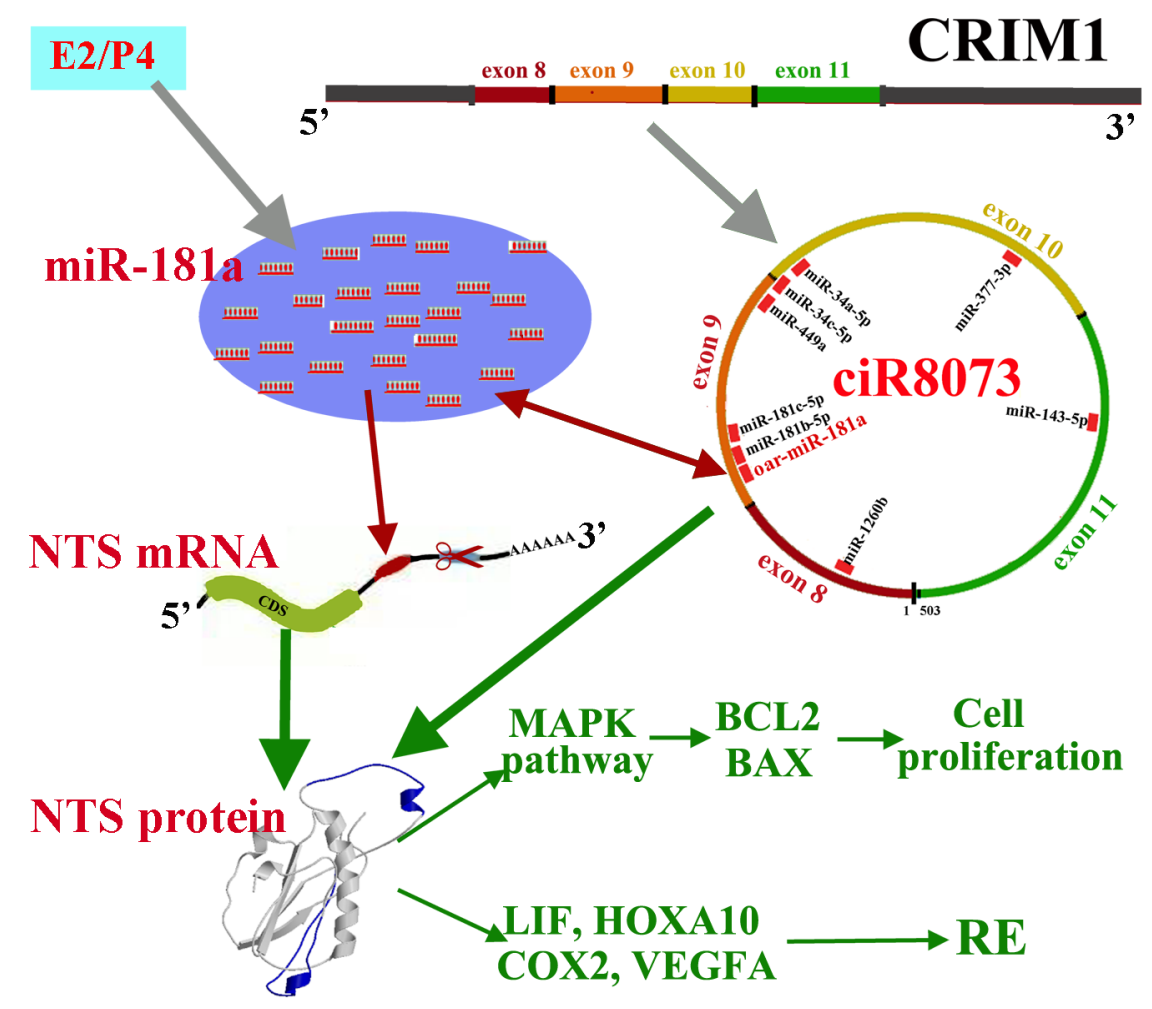

Figure 11: Proposed network of ciR8073-miR181a-NTS in the endometrium of dairy goats. ciR8073 regulates EECs by functioning as a ceRNA, which sequesters miR-181a, thereby relieving its repressive effect on NTS. 
increased through increased SP1 binding to its promoter region, which in turn was mediated through increased SP1 protein levels and decreased inhibitory regulation by the MAPK pathway [60]. In this study, we postulated that NTS decreased SP1, and then decreased P27, activated MAPK pathway, finally inhibited cell apoptosis in EECs in vitro. In addition, many studied showed that PI3K also involved in the process of cell apoptosis [61], thus the PI3K protein level was also detected in this study, and the result showed that NTS decrease it in EECs. All these results suggested that NTS played very important and complex functions in the EECs apoptosis.

In EECs, NTS could inhibit the cells apoptosis by increasing BCL-2 and decreasing BAX. However, it was noteworthy that it also increased Caspase-3, FAS and PTN, decreased SP1 in EECs in vitro. The reasons for this difference might be that NTS prevented EEC apoptosis, what was contrary to the normal processes. Thus, we hypothesized that miR-181a plays a role in the regulation of endometrium cell apoptosis via BCL-2/BAX in EECs of dairy goats.

\section{NTS might participate in the formation of endometrial receptivity}

In this study, NTS could increase some biochemical markers of receptive endometrium, such as LIF, COX2, and HOXA10 in EECs in vitro. However, it should be noted that there was no significant changes on the protein levels of LIF, COX2, and HOXA10 when the NTS was knockdown partly by specific siRNA in EECs. All these results suggested that NTS was helpful to the formation of endometrial receptivity by increasing the protein levels of LIF, COX2, and HOXA10, but this function was not absolutely necessary in EECs in vitro. And more in-depth research was needed to study the functions of NTS in EECs of in dairy goats.

Here we provided direct evidences that ciR8073 could function as a ceRNA, which sequestered miR181a, thereby protecting NTS transcripts from miR181a-mediated suppression (Figure 11). As it was well established that NTS could promote EECs proliferation and increased the expression levels of LIF, COX2, VEGFA and HOXA10, all these data suggestted that ceRNA regulation was of crucial importance in formation of endometrial receptivity in dairy goats. This information will facilitate the further understanding of the ceRNA roles in the regulation mechanism of the RE, and help us to better understand the molecular regulation of endometrial receptivity.

\section{MATERIALS AND METHODS}

\section{Ethics statement}

All animals in this study were maintained according to the No. 5 proclamation of the Ministry of Agriculture,
P. R. China. And animal protocols were approved by the Review Committee for the Use of Animal Subjects of Northwest A\&F University.

\section{Study design and tissue collection}

A total of 20 healthy 24-month-old multiparous dairy goats (Xinong Saanen) were induced to estrous synchronization for this study. The first day of mating was considered to be Day 0 of pregnancy, D5 (PE) and D15 (RE) were important time points for the embryo implantation in goats [62]. The goats were euthanized when the goats lost consciousness caused by intravenous injection of barbiturate $(30 \mathrm{mg} / \mathrm{kg})$ at D5 and D15. Endometrium samples were obtained from the anterior wall of the uterine cavity. All tissue samples were washed briefly with PBS (Phosphate Buffered Saline) and then immediately frozen in liquid nitrogen.

\section{miRNA and transcript differential expression analysis}

miRNA library construction, sequencing, and identification of potential novel miRNAs were showed as before [63]. Expression levels of all of the transcripts, including putative mRNAs, lncRNAs and circRNAs, were quantified as fragments per kilobase of exon per million fragments mapped (FPKM) using the Cuffdiff program from the Cufflinks package [9]. Differential gene expression was determined using Cuffdiff with a p-value of $<0.05$.

\section{Construction of ceRNA network}

The putative miRNA-mRNA, miRNA-lncRNA, miRNA-circRNA interactions were evaluated using the algorithms of Targetscan version 6.2 (http://www. targetscan.org/) and miRanda version 3.3a (http://www. microrna.org/microrna/home.do). The miRNA bindingsite prediction in lncRNAs was based on their full-length sequence in consideration of their non-coding properties. High-confidence miRNA-mRNA/lncRNA/circRNA pairs had a Targetscan context + score percentile $>50$ and miRanda max energy $<-20$. The ceRNA relationships were integrated using an in-house Perl script. The information including all of the above interactions was imported into Cytoscape software version 2.8.0 (http:// www.cytoscape.org) to construct a regulatory network.

\section{Vector construction}

Bioinformatic analysis of miRNA binding sequences in NTS, ciR8073, and ncR915 were performed to identify the target genes of miR-181a using Miranda and Targetscan. To construct reporters for luciferase assays, 3' UTR of NTS, ciR8073 and lncR915 respectively contained the miR-181a target site were cloned and 
inserted downstream of the Renilla luciferase gene in the psiCHECK $^{\mathrm{TM}}-2$ vector (Promega, Madison, USA), and the mutated plasmids were constructed.

To construct reporters for overexpression assays, CDS of NTS, full length of ciR8073 and lncR915 were cloned and inserted in the psiDNA3.1(+) vector (Promega, Madison, USA), and the target site of miR181a were mutated and constructed mutant psiDNA3.1(+)ciR8073(Mu) and psiDNA3.1(+)-lncR915(Mu).

\section{Primary cell culture and purification}

Primary EECs were isolated and purified by digestion of trypsin, centrifugation and difference tempo adherence, and observed by light microscope, and cell purity and identification was evaluated immunocytochemically as previously described [64]. The cells were cultured to the third generation with the same cell culture system to further purify the cells.

\section{Cell transfection}

Mature miR-181a mimics, inhibitors and siRNANTS were synthesized by GenePharma (Shanghai, China). Cells were plated at a density of $7.5 \times 10^{5}$ in 6 -well plates, then the cells were transfected at fifty percent confluency with miR-181a mimics, miR-181a inhibitors, $\mathrm{NC}$, or NC inhibitors at final concentrations of $100 \mathrm{nM}$ using the X-tremeGENE siRNA Transfection Reagent (Roche, Switzerland) according to the manufacturer's concentrations of $100 \mathrm{nM}$ using the X-tremeGENE Lip2000 liposome (Invitrogen, USA).

\section{Cell proliferation and apoptosis assays}

The MTT (Sigma, St. Louis, MO, USA) colorimetric assay was used to screen for cell proliferation as previously described [65]. To further investigate cell proliferation, cell cycle staining Kit was used (Liankebio, Hangzhou, China) according to the manufacturer $i$ instructions. Cell apoptosis analysis was carried out using the Annexin V-FITC/PI apoptosis kit, Annexin-V-positive and PI-negative cells were defined as early apoptotic cells, and the late apoptotic cells were Annexin-V and PI positive cells. Analyses were performed using a flow cytometer (BD Biosciences, San Diego, CA).

\section{Luciferase assay}

The wild-type (psiCHECK2-Wt) or mutated (psiCHECK2-Mu) plasmids were co-transfected with the miR-181a mimic into $293 \mathrm{~T}$ cells, respectively. At 24 h post-transfection, firefly (hluc + ) and Renilla (hRluc) luciferase activities were measured with the Dual-Glo luciferase assay system (Promega, USA). Experiments were performed three times.

\section{RNA extraction and RT-qPCR}

Total RNA was extracted using Trizol reagent (TaKaRa, Dalian, China), RT-qPCR was performed using SYBR Green PCR Master Mix (TaKaRa, Dalian, China) in a $20 \mu \mathrm{l}$ reaction. GAPDH was used as the reference for mRNA and U6 for miR-181a, and all primers for the RTqPCR are shown in Supplementary Table 1. The relative expression levels were calculated using the equation $\mathrm{N}=$ $2^{-\Delta \Delta \mathrm{Ct}}$.

Another total RNA were incubated for $15 \mathrm{~min}$ at $37^{\circ} \mathrm{C}$ with or without (mock) $3 \mathrm{U} / \mu \mathrm{g}$ of RNase $\mathrm{R}$ (Epicenter Biotechnologies, Chicago, USA). To quantify the amount of circRNA, cDNA was synthesized with the Prime Script RT reagent Kit with gDNA Eraser (TaKaRa, Dalian, China) using random hexamers. In particular, the divergent primers annealing at the distal ends of circRNA were used to determine the abundance of circRNA, and the outward-facing primer sets listed in Supplementary Table 1.

\section{Protein extraction and WB analysis}

Protein extraction was performed as described previously [66]. Thirty micrograms of protein from each treatment was used for $12 \%$ SDS-polyacrylamide gel electrophoresis and transferred onto nitrocellulose membranes (Millipore, Bedford, MA, USA) at $100 \mathrm{~V}$ for $1.5 \mathrm{~h}$ in an ice bath. Non-specific binding sites were blocked with 5\% fat-free powdered milk (blocking solution) as well as Tris-buffered saline plus Tween 20 $[0.2 \%(\mathrm{vol} / \mathrm{vol})](\mathrm{TBST})$ at room temperature (RT) for $2 \mathrm{~h}$. After three washes for $10 \mathrm{~min}$ each with TBST, the membranes were incubated overnight at $4{ }^{\circ} \mathrm{C}$ with primary antibodies (as shown in Supplementary Table 2). After this incubation, the membranes were washed three times and then incubated at RT for $2 \mathrm{~h}$ with the horseradish peroxidases (HRP)-conjugated second antibody. After three washes for $5 \mathrm{~min}$ each with TBST, proteins were detected using enhanced chemiluminescence (Advansta, California, USA). Quantification was performed using the Quantity One program (Bio-Rad, California, USA). The experiments were performed three times.

\section{Immunohistochemistry (IHC)}

For IHC, the paraffin-embedded tissue sections were de-paraffinized in xylene for $15 \mathrm{~min}$, and rehydrated in descending concentrations of ethanol (anhydrous ethanol for $5 \mathrm{~min}, 85 \%$ for $5 \mathrm{~min}, 75 \%$ for $5 \mathrm{~min}$ and then rinsed with distilled water). Antigen retrieval in sodium citrate buffer $(\mathrm{pH}=9.0)$ was performed for $10 \mathrm{~min}$ in a microwave oven, then the tissue sections were placed in PBS ( $\mathrm{PH}=7.4)$ and washed three times (each time $5 \mathrm{~min}$ ) on a decoloring shaking bed. Endogenous peroxidases of IHC were inhibited by incubation with 3\% hydrogen peroxide for $25 \mathrm{~min}$ at RT. The samples were blocked 
in $5 \%$ bull serum albumin (BSA) for $30 \mathrm{~min}$, incubated with a primary antibody (as be shown in Supplementary Table 2) at $4^{\circ} \mathrm{C}$ overnight, and then washed three times (each time $5 \mathrm{~min}$ ) on a decoloring shaking bed. Then, the samples were incubated with HRP-labeled secondary antibody for $30 \mathrm{~min}$ at $37^{\circ} \mathrm{C}$. After washing the cells three times in PBS, the color reaction was developed with the substrate diaminobenzidine according to the manufacturer's instructions, and the slides were washed under running water for $5 \mathrm{~min}$ before being counterstained with hematoxylin (Carnaxide, Oeiras, Portugal). After redying the nucleus with hematoxylin and ammonia, the slides were dehydrated with concentrations of ethanol and sealed with xylene for $5 \mathrm{~min}$. The relative density of the positive cells (density/area) in each slide was analyzed using Image-Pro Plus 6.0 Software (Media Cybernetics, USA).

\section{miRNA in situ hybridization (MISH)}

An oligonucleotide probe complementary to the miR-181a sequence was purchased from RiboBio Co., Ltd. (Guangzhou, China). The sequence of the probe was 5e ACTCACCGACAGCGTTGAAGA3TCACCGACAGCGTTGAAGA, China). The sequencedigoxigenin (DIG), and some of these bases were modified with a DIG-labeled LNA (Locked Nucleic Acid). In situ hybridization for miR-181a (MISH) was performed on fixed paraffin-embedded sections as previously described [67]. The relative density of the positive cells (density/area) in each slide was analyzed using Image-Pro Plus 6.0 Software (Media Cybernetics, USA).

\section{Statistical analysis}

All the data were processed with SPSS 17.0 (SPSS Inc., Chicago, IL, USA). One-way ANOVA was used to compare the differences, and the method of the least significant difference (LSD) was used for further analysis. Differences were considered significant when the p-value was $<0.05$ and very significant when $p$-value was $<0.01$.

\section{Abbreviations}

RE, receptive endometrium; PE, pre-receptive endometrium; ceRNA, competing endogenous RNAs; ciR8073, circRNA8073; NTS, Neurotensin; EECs, endometrial epithelium cells; LIF, leukaemia-inhibitory factor; COX2, cyclo-oxygen-ase 2; VEGFA, vascular endothelial growth factor A; HOXA10, homeobox A10; OPN, osteopontin; ncRNAs, non-coding RNA; miRNA, microRNA; lncRNA, long non-coding RNA; circRNA, circular RNA; WOI, window of implantation; MREs, miRNA response elements; DEmiR, differentially expressed miRNA; DEmR, differentially expressed mRNA; DElncR, differentially expressed lncRNA; DEciR, differentially expressed circRNA; FPKM, fragments per kilobase of exon per million fragments mapped; lncR915, lncRNA915; D15, gestational day 15; D5, gestational day 5; hESCs, human endometrial stromal cells; PRL, prolactin; IHC, immunohistochemical; E2, estrogen; $\mathrm{P} 4$, progesterone; UTR, untranslated regions; WB, western blot; p-JNK, phosphorylation JNK; p-MAPK, phosphorylation MAPK; PTN, pleiotrophin; SP1: Sp1 transcription factor; PBS, Phosphate Buffered Saline; MTT, methyl thiazolyl tetrazolium; TBST, Tris-buffered saline plus Tween 20; RT, room temperature; LSD, least significant difference; MISH, miRNA in situ hybridization; FCM, flow cytometry.

\section{Author contributions}

LZ and RXL gathered samples, conceived the report, participated in its design, performed data analysis, interpreted results and drafted the manuscript. BYC, YUS and ZQZ participated in intellectual discussion. YXL, XNM, YZ, XYD and JZL contributed to gathering samples. BYC and YUS designed experiments, analyzed data and wrote the manuscript.

\section{ACKNOWLEDGMENTS}

The authors would like to acknowledge Professor Xiaopeng An for reviewing the manuscript.

\section{CONFLICTS OF INTEREST}

The authors declare no competing financial interest.

\section{FUNDING}

This study was supported by the National Natural Science Foundation of China (31601925), China Postdoctoral Science Foundation (2016T90954 and 2014M552498), Nature Science Foundation of Shaanxi Provincial (2015JM3087), Shaanxi Science and Technology Innovation Project Plan (2015KTCQ03-08 and 2016KTZDNY02-04). The funders had no role in study design, data collection and analysis, decision to publish, or preparation of the manuscript.

\section{REFERENCES}

1. Revel A, Achache H, Stevens J, Smith Y, Reich R. MicroRNAs are associated with human embryo implantation defects. Human reproduction. 2011:der255.

2. Pan Q, Luo X, Toloubeydokhti T, Chegini N. The expression profile of micro-RNA in endometrium and endometriosis and the influence of ovarian steroids on their expression. Mol Human Reprod. 2007; 13:797-806. https:// doi.org/10.1093/molehr/gam063. 
3. Altmäe S, Martinez-Conejero JA, Esteban FJ, Ruiz-Alonso M, Stavreus-Evers A, Horcajadas JA, Salumets A. MicroRNAs miR-30b, miR-30d, and miR-494 regulate human endometrial receptivity. Reproductive Sciences. 2013; 20:308-17.

4. Macklon NS, Stouffer RL, Giudice LC, Fauser BC. The science behind 25 years of ovarian stimulation for in vitro fertilization. Endocrine reviews. 2006; 27:170-207.

5. Shahandeh A. Molecular mechanisms of oncogenic long non-coding RNAs. Bioscience Research. 2013; 10:38-54.

6. Mattick JS, Makunin IV. Non-coding RNA. Human Molecular Genetics. 2006; 15 Spec No 1:R17.

7. Thum T, Condorelli G. Long noncoding RNAs and microRNAs in cardiovascular pathophysiology. Circulation Research. 2015; 116:751.

8. Zhou J, Xiong Q, Chen H, Yang C, Fan Y. Identification of the spinal expression profile of non-coding RNAs involved in neuropathic pain following spared nerve injury by sequence analysis. Frontiers in Molecular Neuroscience. 2017; 10.

9. Wang Y, Xue S, Liu X, Liu H, Hu T, Qiu X, Zhang J, Lei M. Analyses of long non-coding rna and mrna profiling using RNA sequencing during the pre-implantation phases in pig endometrium. Scientific Reports. 2016; 6:20238.

10. Song Y, An X, Zhang L, Fu M, Peng J, Han P, Hou J, Zhou $\mathrm{Z}$, Cao B. Identification and profiling of microRNAs in goat endometrium during embryo implantation. Plos One. 2015; 10:e0122202.

11. Wang Y, Tao H, Wu L, Liu X, Xue S, Lei M. Identification of non-coding and coding RNAs in porcine endometrium. Genomics. 2017; 109:43-50.

12. He Q, Tian L, Jiang H, Zhang J, Li Q, Sun Y, Zhao J, Li $\mathrm{H}$, Liu M. Identification of laryngeal cancer prognostic biomarkers using an inflammatory gene-related, competitive endogenous RNA network. Oncotarget. 2017; 8:9525.

13. Yan B, Yao J, Liu JY, Li XM, Wang XQ, Li YJ, Tao ZF, Song YC, Chen Q, Jiang Q. IncRNA-MIAT regulates microvascular dysfunction by functioning as a competing endogenous RNA. Circulation Research. 2015;116.

14. Ma MZ, Chu BF, Zhang Y, Weng MZ, Qin YY, Gong W, Quan ZW. Long non-coding RNA CCAT1 promotes gallbladder cancer development via negative modulation of miRNA-218-5p. Cell Death \& Disease. 2015; 6:e1583-e.

15. Cesana M, Cacchiarelli D, Legnini I, Santini T, Sthandier O, Chinappi M, Tramontano A, Bozzoni I. A long noncoding RNA controls muscle differentiation by functioning as a competing endogenous RNA. Cell. 2011; 147:358.

16. Hansen TB, Jensen TI, Clausen BH, Bramsen JB, Finsen B, Damgaard CK, Kjems J. Natural RNA circles function as efficient microRNA sponges. Nature. 2013; 495:384-8.

17. Memczak S, Jens M, Elefsinioti A, Torti F, Krueger J, Rybak A, Maier L, Mackowiak SD, Gregersen LH, Munschauer M. Circular RNAs are a large class of animal RNAs with regulatory potency. Nature. 2013; 495:333.
18. Hansen TB, Jensen TI, Clausen BH, Bramsen JB, Finsen B, Damgaard CK, Kjems J. Natural RNA circles function as efficient microRNA sponges. Nature. 2013; 495:384.

19. Li F, Zhang L, Li W, Deng J, Zheng J, An M, Lu J, Zhou Y. Circular RNA ITCH has inhibitory effect on ESCC by suppressing the Wnt/ $\beta$-catenin pathway. Oncotarget. 2015; 6:6001.

20. Zhang L, An XP, Liu XR, Fu MZ, Han P, Peng JY, Hou JX, Zhou ZQ, Cao BY, Song YX. Characterization of the transcriptional complexity of the receptive and prereceptive endometria of dairy goats. Scientific Reports. $2015 ; 5: 14244$.

21. Giorgio AD, Krell J, Harding V, Stebbing J, Castellano L. Emerging roles of competing endogenous RNAs in cancer: Insights from the regulation of PTEN. Molecular \& Cellular Biology. 2013; 33:3976-82.

22. Karreth FA, Pandolfi PP. ceRNA crosstalk in cancer: When ce-bling rivalries go awry. Cancer Discovery. 2013; 3:1113.

23. Thomson DW, Dinger ME. Endogenous microRNA sponges: Evidence and controversy. Nature Reviews Genetics. 2016; 17:272.

24. Sun J, Yan J, Yuan X, Yang R, Dan T, Wang X, Kong G, Gao S. A computationally constructed ceRNA interaction network based on a comparison of the SHEE and SHEEC cell lines. Cellular \& Molecular Biology Letters. 2016; $21: 21$.

25. Ren CE, Zhu X, Li J, Lyle C, Dowdy S, Podratz KC, Byck D, Chen HB, Jiang SW. Microarray analysis on gene regulation by estrogen, progesterone and tamoxifen in human endometrial stromal cells. International Journal of Molecular Sciences. 2015; 16:5864-85.

26. Zhang S, Kong S, Lu J, Wang Q, Chen Y, Wang W, Wang B, Wang H. Deciphering the molecular basis of uterine receptivity. Molecular Reproduction \& Development. 2013; 80:8-21.

27. Su L, Liu R, Cheng W, Zhu M, Li X, Zhao S, Yu M. Expression patterns of micrornas in porcine endometrium and their potential roles in embryo implantation and placentation. Plos One. 2014; 9:e87867.

28. Zhang Q, Zhang H, Jiang Y, Xue B, Diao Z, Ding L, Zhen X, Sun H, Yan G, Hu Y. MicroRNA-181a is involved in the regulation of human endometrial stromal cell decidualization by inhibiting Krüppel-like factor 12. Reprod Biol Endocrinol. 2015; 13:23.

29. Carraway $R$, Leeman SE. The isolation of a new hypotensive peptide, neurotensin, from bovine hypothalami. Journal of Biological Chemistry. 1973; 248:6854.

30. Reinecke M. Neurotensin. Immunohistochemical localization in central and peripheral nervous system and in endocrine cells and its functional role as neurotransmitter and endocrine hormone. Prog Histochem Cytochem. 1985; 16:III,1-VIII, 172. 
31. Sakumoto R, Hayashi KG, Saito S, Kanahara H, Kizaki K, Iga K. Comparison of the global gene expression profiles in the bovine endometrium between summer and autumn. Journal of Reproduction \& Development. 2015; 61:297.

32. Wang K, Liu F, Zhou L-Y, Long B, Yuan S-M, Wang Y, Liu C-Y, Sun T, Zhang X-J, Li P-F. The long noncoding RNA $\mathrm{CHRF}$ regulates cardiac hypertrophy by targeting miR489novelty and significance. Circulation research. 2014; 114:1377-88.

33. Arai M, Yoshioka S, Nishimura R, Okuda K. FAS/FASLmediated cell death in the bovine endometrium. Animal Reproduction Science. 2014; 151:97-104.

34. Otsuki Y. Apoptosis in human endometrium: Apoptotic detection methods and signaling. Medical Electron Microscopy Official Journal of the Clinical Electron Microscopy Society of Japan. 2001; 34:166-73.

35. Heryanto B, Rogers PA. Regulation of endometrial endothelial cell proliferation by oestrogen and progesterone in the ovariectomized mouse. Reproduction. 2002; 123:107-13.

36. Wasowska B, Ludkiewicz B, Stefańczyk-Krzymowska S, Grzegorzewski W, Skipor J. Apoptotic cell death in the porcine endometrium during the oestrous cycle. Acta Veterinaria Hungarica. 2001; 49:71-9.

37. Galán A, O'Connor JE, Valbuena D, Herrer R, Remohí J, Pampfer S, Pellicer A, Simón C. The human blastocyst regulates endometrial epithelial apoptosis in embryonic adhesion. Biology of Reproduction. 2000; 63:430-9.

38. Reis BF, Lima SM, Silva GM, Francisco AM, Barbosa LC, Archangelo SC, Grande RM. Effects of low dose of tibolone on steroid receptors and Bcl-2 on the postmenopausal endometrium. Histology \& Histopathology. 2015; 31: págs. 629-34.

39. Vaskivuo TE, Stenbäck F, Karhumaa P, Risteli J, Dunkel L, Tapanainen JS. Apoptosis and apoptosis-related proteins in human endometrium. Molecular \& Cellular Endocrinology. 2000; 165:75-83

40. Liu G, Li Y, Gao X. MicroRNA-181a is upregulated in human atherosclerosis plaques and involves in the oxidative stress-induced endothelial cell dysfunction through direct targeting Bcl-2. european review for medical and pharmacological sciences. 2016; 20:3092-100.

41. Liu CQ, Yuan Y, Wang ZX. Effects of leukaemia inhibitory factor on endometrial receptivity and its hormonal regulation in rabbits. Cell Biology International. 2001; 25:1029-32.

42. Miravetvalenciano JA, Rinconbertolin A, Vilella F, Simon C. Understanding and improving endometrial receptivity. Current Opinion in Obstetrics \& Gynecology. 2015; 27:187.

43. Isayama K, Zhao L, Chen H, Yamauchi N, Shigeyoshi Y, Hashimoto S, Hattori MA. Removal of Rev-erb $\alpha$ inhibition contributes to the prostaglandin $\mathrm{G} / \mathrm{H}$ synthase 2 expression in rat endometrial stromal cells. Ajp Endocrinology \& Metabolism. 2015; 308:2462-7.
44. Das SK, Chakraborty I, Wang J, Dey SK, Hoffman LH. Expression of vascular endothelial growth factor (VEGF) and VEGF-receptor messenger ribonucleic acids in the periimplantation rabbit uterus. Biology of Reproduction. 1997; 56:1390-9.

45. Chakraborty I, Das SK, Dey SK. Differential expression of vascular endothelial growth factor and its receptor mRNAs in the mouse uterus around the time of implantation. Journal of Endocrinology. 1995; 147:339-52.

46. Demir R, Yaba A, Huppertz B. Vasculogenesis and angiogenesis in the endometrium during menstrual cycle and implantation. Acta Histochemica. 2010; 112:203-14.

47. Zanatta A, Rocha AM, Carvalho FM, Pereira RMA, Taylor HS, Motta ELA, Baracat EC, Serafini PC. The role of the Hoxa10/HOXA10 gene in the etiology of endometriosis and its related infertility: A review. Journal of Assisted Reproduction and Genetics. 2010; 27:701-10.

48. Petracco R, Grechukhina O, Popkhadze S, Massasa E, Zhou Y, Taylor HS. MicroRNA 135 regulates HOXA10 expression in endometriosis. Journal of Clinical Endocrinology \& Metabolism. 2011; 96:1925-33.

49. Horcajadas JA, Anne R, Francisco D, Ana C, Antonio P, Carlos S. Determinants of endometrial receptivity. Annals of the New York Academy of Sciences. 2005; 1034:166-75.

50. Kontovounisios C, Qiu S, Rasheed S, Darzi A, Tekkis P. The role of neurotensin as a novel biomarker in the endoscopic screening of high-risk population for developing colorectal neoplasia. updates in surgery. 2017.

51. Jing L, Song J, Zaytseva YY, Liu Y, Rychahou P, Kai J, Starr ME, Ji TK, Harris JW, Yiannikouris FB. An obligatory role for neurotensin in high-fat-diet-induced obesity. Nature. 2016; 533:411.

52. Liu D, Xu W, Ding X, Yang Y, Lu Y, Fei K, Su B. Caspase 8 polymorphisms contribute to the prognosis of advanced lung adenocarcinoma patients after platinum-based chemotherapy. Cancer Biology \& Therapy. 2017.

53. Arai M, Yoshioka S, Tasaki Y, Okuda K. Remodeling of bovine endometrium throughout the estrous cycle. Animal Reproduction Science. 2013; 142:1-9.

54. Harada T, Kaponis A, Iwabe T, Taniguchi F, Makrydimas G, Sofikitis N, Paschopoulos M, Paraskevaidis E, Terakawa N. Apoptosis in human endometrium and endometriosis. Human Reproduction Update. 2004; 10:29-38.

55. Iwahori S, Yasui Y, Kudoh A, Sato Y, Nakayama S, Murata $\mathrm{T}$, Isomura $\mathrm{H}$, Tsurumi T. Identification of phosphorylation sites on transcription factor $\mathrm{Sp} 1$ in response to DNA damage and its accumulation at damaged sites. Cellular Signalling. 2008; 20:1795.

56. Liguo, Zhao, Wantong, Meining, niuliang, cheng. silencing Sp1 suppresses telomerase activity and promotes apoptosis of SW480 cells line in colorectal carcinoma. 中德临床肿 瘤学杂志. 2011; 10:220-4. 
57. Muramatsu T. Midkine and pleiotrophin: Two related proteins involved in development, survival, inflammation and tumorigenesis. The Journal of Biochemistry. 2002; 132:359-71.

58. Mansouriattia N, Aubert J, Reinaud P, Girauddelville C, Taghouti G, Galio L, Everts RE, Degrelle S, Richard C, Hue I. Gene expression profiles of bovine caruncular and intercaruncular endometrium at implantation. Physiological Genomics. 2009; 39:14-27.

59. Chen J, Xu J, Li Y, Zhang J, Chen H, Lu J, Wang Z, Zhao X, $\mathrm{Xu} \mathrm{K}$, Li Y. Competing endogenous RNA network analysis identifies critical genes among the different breast cancer subtypes. Oncotarget. 2016.

60. Chen F, Kim E, Wang CC, Harrison LE. Ciglitazoneinduced p27 gene transcriptional activity is mediated through $\mathrm{Sp} 1$ and is negatively regulated by the MAPK signaling pathway. Cellular Signalling. 2005; 17:1572-7.

61. Pan BS, Wang YK, Lai MS, Mu YF, Huang BM. Cordycepin induced MA-10 mouse leydig tumor cell apoptosis by regulating p38 mapks and $\mathrm{PI} 3 \mathrm{~K} / \mathrm{AKT}$ signaling pathways. Scientific Reports. 2017; 108:S32.

62. Igwebuike U. A review of uterine structural modifications that influence conceptus implantation and development in sheep and goats. Animal reproduction science. 2009; 112:1-7.
63. Song Y, An X, Zhang L, Fu M, Peng J, Han P, Hou J, Zhou $\mathrm{Z}$, Cao B. Identification and profiling of microRNAs in goat endometrium during embryo implantation. Plos One. 2015; 10.

64. Pierro E, Minici F, Alesiani O, Miceli F, Proto C, Screpanti I, Mancuso S, Lanzone A. Stromal-epithelial interactions modulate estrogen responsiveness in normal human endometrium1. Biology of Reproduction. 2001; 64:831-8.

65. Sun X, He Y, Ma T-T, Huang C, Zhang L, Li J. Participation of miR-200a in TGF- $\beta 1$-mediated hepatic stellate cell activation. Molecular and cellular biochemistry. 2014; 388:11-23.

66. Subramaniam KS, Omar IS, Kwong SC, Mohamed Z, Woo YL, Mat Adenan NA, Chung I. Cancer-associated fibroblasts promote endometrial cancer growth via activation of interleukin-6/STAT-3/c-Myc pathway. American Journal of Cancer Research. 2016; 6:200-13.

67. Li J, Yang H, Li Y, Liu Y, Chen S, Qi C, Zhang Q, Lan T, He X, Guan XY. microRNA-146 up-regulation predicts the prognosis of non-small cell lung cancer by miRNA in situ hybridization. Experimental \& Molecular Pathology. 2014; 96:195-9. 